\title{
Electron Transfer to the Trinuclear Copper Cluster in Electrocatalysis by the Multicopper Oxidases
}

\author{
Alina Sekretareva, ${ }^{\dagger} \#, *$ Shiliang Tian, ${ }^{\dagger}$ Sébastien Gounel, ${ }^{\|}$Nicolas Mano,,$\AA \|$ \\ Edward I. Solomon ${ }^{\dagger, \ddagger * *}$ \\ ${ }^{\dagger}$ Department of Chemistry, Stanford University, Stanford, California 94305, United States \\ $¥$ SLAC National Accelerator Laboratory, Stanford University, California 94025, United States \\ \# Department of Chemistry, Ångström Laboratory, Uppsala University, SE-75120, Uppsala, \\ Sweden \\ $\S$ CNRS, CRPP, UPR 8641, 33600 Pessac, France \\ " Université de Bordeaux, CRPP, UMR5031, 33600 Pessac, France
}

\begin{abstract}
High-potential multicopper oxidases (MCOs) are excellent catalysts able to perform the oxygen reduction reaction (ORR) at remarkably low overpotentials. Moreover, MCOs are able to interact directly with the electrode surfaces via direct electron transfer (DET), that makes them the most commonly used electrocatalysts for oxygen reduction in biofuel cells. The central question in MCO electrocatalysis is whether the type $1(\mathrm{~T} 1) \mathrm{Cu}$ is the primary electron acceptor site from the electrode, or whether electrons can be transferred directly to the trinuclear copper cluster (TNC), bypassing the rate-limiting intramolecular electron transfer step from the T1 site. Here, using sitedirected mutagenesis and electrochemical methods combined with data modeling of electrode kinetics we have found that there is no preferential superexchange pathway for DET to the T1 site. However, due to the high reorganization energy of the fully oxidized TNC, electron transfer from the electrode to the TNC does occur primarily through the T1 site. We have further demonstrated that the lower reorganization energy of the TNC in its two-electron reduced, alternative resting, form enables DET to the TNC, but this only occurs in the first turnover. This study provides insight into the factors that control the kinetics of electrocatalysis by the MCOs and a guide for the design of more efficient biocathodes for the ORR.
\end{abstract}




\section{INTRODUCTION-}

Multicopper oxidases (MCOs) comprise a family of enzymes that catalyze the oxidation of various substrates concomitant with the oxygen reduction reaction (ORR). ${ }^{1,2}$ This reaction in all MCOs is catalyzed by a minimum of four copper ions, which form the active site of the enzyme. Substrates are oxidized at the solvent-accessible mononuclear type $1 \mathrm{Cu}$ site (T1), and the electrons are then transferred through the protein via intramolecular electron transfer over a distance of $\sim 13 \AA$ to a trinuclear $\mathrm{Cu}$ cluster (TNC). The TNC is comprised of one type 2 (T2) $\mathrm{Cu}$ and a type 3(T3) $\mathrm{Cu}$ pair, where the four-electron reduction of dioxygen occurs. ${ }^{1,3}$ One of the key characteristics of the MCOs is the standard redox potential of their $\mathrm{T} 1 \mathrm{Cu}$, which varies widely from as low as $\sim 300 \mathrm{mV}$ to as high as $\sim 800 \mathrm{mV}$ vs. normal hydrogen electrode (NHE) for different MCOs and this determines the overpotential of the ORR. ${ }^{4,5}$ The overpotential required for the ORR is remarkably small in the high potential MCOs, leading to their importance as heterogeneous catalysts for constructing efficient biocathodes in fuel cells. 6,7

The mechanism of homogeneous catalysis in MCOs, including high potential MCOs, has been well defined through spectroscopic and computational studies. ${ }^{8-12}$ In the catalytic cycle, the $\mathrm{T} 1$ site is the primary electron acceptor which receives electrons from the reducing substrates and transfers them to the TNC through a Cys-His superexchange pathway. ${ }^{13}$ The TNC in the fully reduced state reacts with $\mathrm{O}_{2}$ to form the peroxy intermediate (PI), in which $\mathrm{O}_{2}$ is reduced by two electrons and is coordinated by all three $\mathrm{Cu}$ 's of the $\mathrm{TNC}$, where the $\mathrm{T} 2 \mathrm{Cu}$ and one of $\mathrm{T} 3 \mathrm{Cu}$ 's are oxidized. ${ }^{8,12,14}$ Reduction of this intermediate by two more electrons leads to the fully oxidized native intermediate $(\mathrm{NI})$, that is converted back to the all-cuprous state by intramolecular electron transfer from the T1 site to close the catalytic cycle. ${ }^{9,15}$ In the absence of additional electrons NI 
decays to the resting fully oxidized state (RO), that is not relevant for the catalytic cycle, except for the slow ferroxidases, Fet3p, and ceruloplasmin. ${ }^{15-17}$

Halides, $\mathrm{F}^{-}$and $\mathrm{Cl}^{-}$, are known inhibitors of MCOs in solution. It has been demonstrated that fluoride has a high affinity for the fully oxidized TNC, binding in the center of the TNC to the T2 site along its open equatorial coordination position trans to a hydroxide ligand. ${ }^{18}$ Fluoride binding reduces the redox potential of the TNC by $150-200 \mathrm{mV}$ as determined by potentiometric titrations and computational studies. ${ }^{19-21}$ Addition of chloride to the fully oxidized enzyme results in a two-electron reduced TNC. This form, referred to as 'alternative resting' (AR), possesses distinct spectroscopic features compared to the RO form. ${ }^{22}$ In the RO form, the $\mathrm{T} 2$ site has 'normal' electron paramagnetic resonance (EPR) parameters of $\mathrm{g}_{\|}=2.22-2.27$ and $A_{\|}=170-200 \times 10^{-4} \mathrm{~cm}^{-1}$, and the absence of intense UV-Vis absorption features. The T3 $\mathrm{Cu}(\mathrm{II})$ 's are antiferromagnetically coupled through a hydroxide bridge, with intense $\mu-\mathrm{OH}$ charge-transfer transitions around $330 \mathrm{~nm}$. The distance between the T3 Cu's is $<4 \AA .{ }^{1}$ In the AR form, the single $\mathrm{Cu}(\mathrm{II})$ of the TNC shows a large $\mathrm{g}_{\|}$and small $\mathrm{A}_{\|}\left(80-100 \times 10^{-4} \mathrm{~cm}^{-1}\right)$, lacks the $330 \mathrm{~nm}$ absorption intensity, and has a T3 $\mathrm{Cu}-\mathrm{Cu}$ distance of $>4.7 \AA{ }^{22}$

The mechanism of heterogeneous catalysis by the MCOs, particularly for proteins coupled to an electrode through direct electron transfer (DET), is less established. ${ }^{5,6}$ The central question in $\mathrm{MCO}$ electrocatalysis is whether the $\mathrm{T} 1$ site is the primary acceptor site similar to the mechanism in homogeneous catalysis, or whether electrons can be transferred directly to the TNC, bypassing intramolecular electron transfer from the T1 site. According to the currently accepted model of electrocatalysis, the potential of the redox center that accepts/donates electrons to/from the electrode controls catalysis and not the potential of the actual catalytic site. ${ }^{23}$ Many studies have reported a correlation between the onset ORR potential and the potential of the T1 site, which 
implies DET through the T1 site. ${ }^{5,24,25}$ The mechanism of electrocatalysis with electron transfer via the $\mathrm{T} 1$ site should be similar to well-characterized homogeneous catalysis, with the only difference being that the electrode replaces the reducing substrate as an electron source.

However, in several reports, the onset of the ORR catalyzed by immobilized high potentials MCOs was observed at low potentials. The catalytic onset at $\sim 300 \mathrm{mV}$ vs. NHE was reported for Trametes hirsuta laccase $\left(\mathrm{E}^{0^{\prime}}=790 \mathrm{mV}\right.$ vs. NHE) ${ }^{4}$ immobilized on bare and self-assembled monolayer modified gold electrodes. ${ }^{26,27}$ ORR catalyzed by Myrothecium verrucaria bilirubin oxidase $\left(\mathrm{E}^{0^{\prime}}=570 \mathrm{mV}\right.$ vs. NHE) ${ }^{24}$ at $\sim 450 \mathrm{mV}$ vs. NHE was detected on gold electrodes modified with self-assembled monolayers. ${ }^{28}$ The low-potential wave was attributed to the redox potential of the electron acceptor site in the TNC, which was assumed to be the site for DET from the electrode. Inhibition of MCOs immobilized on electrodes by halides has been reported. ${ }^{29-32}$ Observed potential shifts of the onset ORR to lower redox potentials upon fluoride additions had been attributed to DET to the fluoride-bound TNC. ${ }^{31}$ In the case of $\mathrm{Cl}^{-}$inhibition, it was earlier suggested that this anion binds near to the T1 site reducing its redox potential. ${ }^{32}$ Recently, it was hypothesized that the TNC in the AR state formed upon $\mathrm{Cl}^{-}$addition can directly couple to the electrode. ${ }^{30}$ Overall, experimental observations of catalytic waves in high potential MCOs at low potentials led to speculations suggesting the possibility of DET to the TNC. We have demonstrated previously that the first electron transfer step from the $\mathrm{T} 1$ site to the TNC in reduction of NI is the rate-limiting step in the homogeneous ORR by high potential MCOs, determining the overall turnover frequency and efficiency of the catalysis. ${ }^{11}$ DET to the TNC bypassing the rate-limiting step, thus, will significantly alter the mechanism of ORR catalysis.

Discrepancies in the literature on whether the T1 site or the TNC directly couple to the electrode in the electrocatalytic ORR led us to systematically study the DET process between the 
electrode and the high potential MCOs. Herein, using electrochemical methods, we investigate electrocatalysis by two T1 variants: M468Q Magnaporthe oryzae bilirubin oxidase (M468Q MoBOD) and T1-depleted Bacillus pumilus bilirubin oxidase (T1D BpBOD) in comparison to these wild type proteins. We first characterize these mutants spectroscopically by EPR and UVVis spectroscopies to ensure that T1 mutations do not perturb the TNC site. We also determine the $\mathrm{O}_{2}$ reaction kinetics of the variants in solution by stopped-flow absorption and Clark-electrode measurements. We then perform protein film electrochemistry measurements (cyclic voltammetry (CV) and linear sweep voltammetry (LSV)) on the variants and the WT proteins in their RO forms and after additions of $\mathrm{F}^{-}$and $\mathrm{Cl}^{-}$and develop a mathematical model to quantitatively describe the cathodic process. Our model accounts for all experimental data by introducing terms describing the electronic coupling to and the reorganization energies of the primary electron acceptor sites. These studies provide insight into factors controlling which site primarily couples with the electrode via DET in electrocatalysis by MCOs.

\section{EXPERIMENTAL SECTION}

\section{Chemicals and Materials}

All chemicals were reagent grade and used without further purification. Water was purified to a resistivity of $>17 \mathrm{M} \Omega \mathrm{cm}$ using a Barnstead Nanopure deionizing system.

\section{Protein Expression, Purification and Characterization}

The integration of BOD (Genbank accession EDJ95889.1) from Magnaporthe oryzae (ATCC MYA-4617) in pPicZ $\alpha a^{33}$ and the insertion of the ORF coding from BOD from Bacillus pumilus (accession no. A8FAG9) into a pET21a vector was made as previously described. ${ }^{34}$ All 
mutations were made with the quick-change II site-directed mutagenesis kit (Agilent- USA) with the following two primers:

\begin{tabular}{|l|l|}
\hline Mo BOD M468Q forward & CACGAGGACCACGACCAGATGGCCGCCTTCAAC \\
\hline Mo BOD M468Q reverse & GTTGAAGGCGGCCATCTGGTCGTGGTCCTCGTG \\
\hline Mo BOD T1D forward & GTTACATGTTCCACTCTCACAACCTGATCCAC \\
\hline Mo BOD T1D reverse & GTGGATCAGGTTGTGAGAGTGGAACATGTAAAC \\
\hline Bp BOD M502Q forward & TTAGAGGATTATGACCAGATGAGGCCGATGGAT \\
\hline Bp BOD M502Q reverse & ATCCATCGGCCTCATCTGGTCATAATCCTCTAA \\
\hline Bp BOD T1D forward & AGGTACGTGTGGCATTCTCATATATTAGAGCAC \\
\hline Bp BOD T1D reverse & GTGCTCTAATATATGAGAATGCCACACGTACCT \\
\hline
\end{tabular}

The presence of the mutations was verified by DNA sequencing (Genewiz, Germany). All the other steps were performed as previously described for BOD from $M$. oryzae ${ }^{33}$ and for BOD from B. pumilus. ${ }^{35}$

The enzyme activity was determined spectrophotometrically at $37{ }^{\circ} \mathrm{C}$ by following the oxidation of $1 \mathrm{mM}$ ABTS at $420 \mathrm{~nm}\left(\varepsilon 420 \mathrm{~nm}=0,36 \mathrm{mM}^{-1} \mathrm{~cm}^{-1}\right)$ in a $0.1 \mathrm{M}$ Mcllvaine citratephosphate buffer, $\mathrm{pH} 4$. One unit was defined as the amount of enzyme that oxidized $1 \mu \mathrm{mol}$ of substrate per minute.

Total copper and $\mathrm{Cu}(\mathrm{I})$ content were determined by the biquinoline method. ${ }^{36} \mathrm{Cu}(\mathrm{I})$ quantitation was performed under anaerobic conditions.

\section{Sample Preparation}

All samples were prepared in Mcllvaine buffer (100 mM to $200 \mathrm{mM}$ citrate-phosphate buffer) at $\mathrm{pH}$ 5.0. The fully oxidized enzyme was obtained by reoxidation of the fully reduced protein by exposure to oxygen. Solutions of fully reduced protein were prepared in the glovebox maintained under $\mathrm{N}_{2}$ atmosphere by reduction of the anaerobic solution of as-isolated protein by 1.5-2.0 times excess of sodium dithionite. To enable a fast complete reduction of the T1D mutant, 
excess methyl viologen was added together with sodium dithionite. The remaining reductants were removed by size exclusion chromatography using PD-10 desalting columns (GE Healthcare).

\section{EPR and UV-Vis Spectroscopy}

UV-Visible (UV-Vis) absorption spectra were acquired on a Hewlett-Packard HP8452A diode array in a $1 \mathrm{~cm}$ path length cuvette. All X-band spectra were obtained with a Bruker EMX spectrometer, ER 041 XG microwave bridge, with either an ER4119HS or ER4116DM cavity. Sample temperatures of $77 \mathrm{~K}$ were maintained using a liquid $\mathrm{N}_{2}$ cold finger dewar. The concentration of paramagnetic coppers was determined from spin quantification of EPR spectra using $0.945 \mathrm{mM} \mathrm{CuSO}_{4}$ in 40 vol \% glycerol as a standard. ${ }^{37}$ EPR spectra simulation was performed using the EasySpin MATLAB toolbox. ${ }^{38}$

\section{Stopped-flow Spectroscopy}

For stopped-flow measurements, the reaction solutions were loaded into gastight syringes (Hamilton) inside the glovebox maintained under the $\mathrm{N}_{2}$ atmosphere and transferred using threeway valves to exclude air. A typical protein concentration for stopped-flow experiments was $100 \mu \mathrm{M}$ before mixing. Kinetic data with $\sim 2 \mathrm{~ms}$ dead time were collected using an Applied Photophysics SX.19 stopped-flow absorption spectrometer equipped with a $\mathrm{Hg} / \mathrm{Xe}$ Arc lamp and outfitted with polyetheretherketone tubing. The spectrophotometer was placed in an anaerobic purge box (Cleatech custom glovebox) kept under an Ar atmosphere and equipment with an oxygen sensor (Neutronics Model 1100). All experiments were performed at $23{ }^{\circ} \mathrm{C}$ with the temperature maintained using a water temperature bath (Fisher Scientific Isotherm 3016). Kinetic traces were fit using the Origin 2018 (Academic) software package.

\section{Homogeneous Catalytic Activity Measurements}


Enzyme activity monitoring under multiple turnover conditions was performed with the Clarktype $\mathrm{O}_{2}$ microsensor (Unisense). The Clark electrode was first calibrated in degassed $\left(0 \mu \mathrm{M} \mathrm{O}_{2}\right)$, air-saturated $\left(250 \mu \mathrm{M} \mathrm{O}_{2}\right)$, and oxygen-saturated $\left(1.2 \mathrm{mM} \mathrm{O}_{2}\right)$ Mcllvaine buffer $(\mathrm{pH} 5.0)$ at $23{ }^{\circ} \mathrm{C}$. Protein solution (either in the fully oxidized or the fully reduced form) was added to a gas-tight vial with ABTS solution in air-saturated Mcllvaine buffer and oxygen consumption was monitored for $\sim 300 \mathrm{~s}$.

\section{Potentiometric Titrations}

The reduction potential of the M468Q MoBOD T1 Cu site was obtained by the poised potential method reported previously. ${ }^{39}$ Briefly, $40 \mu \mathrm{l}$ of degassed $500 \mathrm{mM} \mathrm{K}_{3}\left[\mathrm{Fe}(\mathrm{CN})_{6}\right]$ stock solution were added to $2000 \mu \mathrm{L}$ of degassed $10 \mu \mathrm{M}$ solution of fully oxidized M468Q MoBOD in a $1 \mathrm{~cm}$ path length anaerobic cuvette with a Teflon stopcock to yield a final concentration of $10 \mathrm{mM}$ $\mathrm{K}_{3}\left[\mathrm{Fe}(\mathrm{CN})_{6}\right]$. Small quantities of concentrated stock solution of degassed $\mathrm{K}_{4}\left[\mathrm{Fe}(\mathrm{CN})_{6}\right]$ were added under stirring to yield a $\mathrm{K}_{3}\left[\mathrm{Fe}(\mathrm{CN})_{6}\right] / \mathrm{K}_{4}\left[\mathrm{Fe}(\mathrm{CN})_{6}\right]$ ratios of $0.1,0.2,0.5,1.0,2.0,5.0,10,20$, and 50. The absorption spectra were taken every minute until the reaction was completed (>30 min). All solutions were prepared in the glovebox.

\section{Protein-Film Electrochemistry}

Electrochemical experiments were performed with a PINE potentiostat/ galvanostat model WaveNow (Pine Research Instrumentation), using an $\mathrm{Ag} / \mathrm{AgCl}(3 \mathrm{M} \mathrm{KCl})$ reference and a platinum wire as counter electrodes. The working electrodes were spectrographic graphite electrodes (SPGE) from RingsdorffWerke GmbH, Bonn, Germany, (type RW001, 3.05 mm diameter and 13\% porosity, http://www.sglcarbon.com). SPGEs were prepared by wet polishing the end of a rod using waterproof emery paper. The electrodes were then rinsed with Milli-Q water and allowed 
to dry in the air. The electrodes were then modified with carbon nanofibers (CNFs). CNFs were suspended in DMF to obtain a final solution with a concentration of $4 \mathrm{mg} / \mathrm{mL}$. The solution was sonicated for $30 \mathrm{~min} .5 \mu \mathrm{l}$ of the solution was applied onto the SPGE electrode surface and left to dry at $60^{\circ} \mathrm{C}$. Subsequently, $5 \mu \mathrm{L}$ of enzyme solution $(\sim 0.2 \mathrm{mM})$ was placed on top of the polished rod, and adsorption was allowed to occur. After 15 min the electrode was rinsed with Milli-Q water, inserted in a Teflon holder, and used in the electrochemical cell.

The onset potentials were determined as potentials at which catalytic currents start to deviate (the catalytic current exceeds $3 \times$ standard deviation of the noise level) from non-catalytic currents recorded in deoxygenated buffer.

\section{RESULTS}

\section{Spectroscopic Characterization of Type 1 Variants of High Potential Bilirubin Oxidases}

Two coordinating residues of the $\mathrm{T} 1 \mathrm{Cu}$ site of the high potential MoBOD and BpBOD were mutated to perturb the electron transfer to the TNC. In the first variant, M468Q MoBOD, the axial Met ligand of the T1 site was replaced by Gln. In the second variant, the T1 Cu-coordinating Cys was replaced by Ser, resulting in a T1-depleted (T1D) BpBOD derivative. (Note that the T1D variant of MoBOD was not stable probably due to issues with Cu loading at the TNC (Figure S1), and the M-to-Q variant of BpBOD did not express). From a biquinoline assay, the as-isolated M468Q mutant loads approximately four coppers, while the T1D variant loads three coppers per protein, verifying that $\mathrm{Cu}$ is not bound at the T1 site in T1D (Table 1). This correlates with the lack of the $610 \mathrm{~nm} \mathrm{T1}$ absorbance feature in the spectrum of T1D (Figure 1F). The EPR spectrum of the T1D variant (Figure 1C, blue) also lacks the T1 signal and exhibits a typical tetragonal copper T2 Cu(II) spectrum, which can be simulated with 'normal' T2 Cu(II) spin Hamiltonian 
parameters (Table 2; Figure 1C, cyan), similar to those earlier reported for T1D Fet3p ${ }^{40,41}$ and T1Hg Rhus vernificera laccase, ${ }^{42}$ indicating that the T1 mutation did not affect the TNC. Spin quantitation of the EPR spectrum of the T1D variant shows that there are 1.1 spins/protein (Table 1), consistent with the absence of the paramagnetic $\mathrm{T} 1 \mathrm{Cu}$.

Earlier, we found that the primary form of as-isolated BOD is the AR derivative, where two of the three $\mathrm{Cu}$ atoms in the TNC were reduced. The RO (the fully oxidized TNC) and the AR forms of WT BOD can be interconverted. ${ }^{22,43}$ Thus, complete anaerobic reduction of the AR form of the enzyme by dithionite with subsequent exposure to dioxygen results in the fully oxidized protein in the $\mathrm{RO}$ form. When the fully oxidized enzyme is exposed to an excess of a $\mathrm{Cl}^{-}$ion, regeneration of the AR form occurs. ${ }^{22} \mathrm{RO}$ and $\mathrm{AR}$ show distinct spectroscopic features that can be easily distinguished using a combination of Abs and EPR spectra (Figure 1A and D, blue vs. red spectra). Specifically, the two-electron reduced AR TNC has characteristic spin Hamiltonian parameters of the non-T1 $\mathrm{Cu}(\mathrm{II})$ that are distinct from those for the 'normal' $\mathrm{T} 2 \mathrm{Cu}(\mathrm{II})$ (i.e. a large $\mathrm{g}_{\|}$and small $\mathrm{A}_{\|}$) and lacks the $330 \mathrm{~nm}$ absorption intensity. ${ }^{20}$ The EPR spectrum of the fully oxidized WT (Figure 1A, blue line) was simulated using the parameters obtained for the T2 $\mathrm{Cu}$ (II) in T1D and allowing the $\mathrm{T} 1 \mathrm{Cu}(\mathrm{II})$ spin Hamiltonian parameters to adjust. The resulting values for the $\mathrm{T} 1 \mathrm{Cu}$ (II) give the small parallel hyperfine coupling constant of $\mathrm{A}_{\|} \sim 70 \times 10^{-4} \mathrm{~cm}^{-1}$, typical for blue copper sites and similar to those reported for high potential BODs. ${ }^{1,22}$ Simulation of the spectrum of the WT MoBOD after additions of $\mathrm{Cl}^{-}$(Figure 1A, red line) using the $\mathrm{T} 1 \mathrm{Cu}$ (II) spin Hamiltonian parameters of the RO form and letting the non-T1 copper parameters adjust gives values consistent with the AR form (high $g_{z}$ and small $A_{z}$, Table 2).

EPR and Abs spectra of the M468Q mutant in the fully oxidized form are shown in Figures 1B and E, respectively. The M468Q mutant in the fully oxidized form has four $\mathrm{Cu}$ per protein, 
none of which are in the reduced, $\mathrm{Cu}(\mathrm{I})$ form (Table 1). The EPR spectrum of the fully oxidized M468Q (Figure 1B, blue) with a spin-integrated intensity of $1.7 \mathrm{Cu}$ (II) 's per molecule, displays a 'normal' $\mathrm{T} 2 \mathrm{Cu}(\mathrm{II}) \mathrm{EPR}$ spectrum, similar to the $\mathrm{T} 2 \mathrm{Cu}(\mathrm{II})$ signal in the $\mathrm{RO}$ form of the WT protein and a perturbed $\mathrm{T} 1 \mathrm{Cu}(\mathrm{II})$ signal (T1' in Figure 1B) due to the axial ligand variation. From ligandfield theory, the EPR g values are proportional to the amount of metal character in the halfoccupied HOMO (i.e., covalency) and inversely proportional to the ligand-field transition energies. Mutation of the axial Met to Gln leads to a shift of the T1 Cu out of the Cys(His)2 plane resulting in a decrease of the ligand-field transitions, and therefore, an increase of $g_{z}$ and an associated decrease of the $\mathrm{A}_{\mathrm{z}}$ value due to the $\mathrm{g}$ value effect on the orbital dipolar contribution to the hyperfine coupling. ${ }^{44}$ The observed changes of the T1 Cu spin-Hamiltonian parameters in M468Q compared to WT MoBOD (Table 2) are consistent with this prediction and the changes earlier reported in the same variants for Myrothecium verrucaria BOD, ${ }^{45}$ stellacyanin, ${ }^{44}$ rusticyanin, ${ }^{46}$ plastocyanin, ${ }^{47}$ and azurin. ${ }^{48}$ The Abs spectrum of the M468Q in the fully oxidized form (Figure 1E, blue) shows a $330 \mathrm{~nm}$ shoulder, consistent with the RO form of the TNC cluster. The broad T1 Abs band at around $750 \mathrm{~nm}$ observed in WT in Figure 1D (a ligand field transition with significant charge transfer mixing) is shifted towards $850 \mathrm{~nm}$ and has lower intensity in M468Q. There is also an additional small band in the 400-500 $\mathrm{nm}$ region. The Abs and EPR spectral changes of the M468Q variant resemble those reported for the M467Q mutant of Myrothecium verrucaria BOD. ${ }^{45}$

The EPR and Abs spectra of the M468Q mutant after addition of $\mathrm{Cl}^{-}$are shown in Figures $1 \mathrm{~B}$ and $1 \mathrm{E}$, respectively (red curves). We initially anticipated that the $\mathrm{T} 1$ mutation would not affect the TNC, so it should be possible to simulate the spectrum of M468Q after addition of $\mathrm{Cl}^{-}$(Figure $1 \mathrm{~B}$, red line) using the parameters of the $\mathrm{T} 1$ ' site obtained for the fully oxidized protein and the 
AR parameters for the non-T1 copper. However, this did not reproduce the experimental spectrum. So, the parameters of the non- $\mathrm{T} 1 \mathrm{Cu}$ were allowed to float to reproduce the experimental data. The resulting spin Hamiltonian parameters of the non- $\mathrm{T} 1 \mathrm{Cu}$ differ from the AR parameters of WT MoBOD (Table 2) and are similar to those of a typical tetragonal copper center. But from $\mathrm{Cu}(\mathrm{I})$ quantitation using an anaerobic biquinoline assay M468Q MoBOD incubated with excess $\mathrm{Cl}^{-}$has 2.2 reduced Cu's (Table 1). The results of the copper quantification and the disappearance of the $330 \mathrm{~nm}$ shoulder in the Abs spectrum (Figure 1E, red) are consistent with the formation of the twoelectron reduced TNC, which we denote as AR'. The difference in the EPR spectra of the AR (WT) and AR'(M468Q) forms indicates that the T1 site mutation affects the TNC in the AR state.

\section{Kinetic Characterization of Type 1 Variants of High Potential Bilirubin Oxidases in}

\section{Solution}

We investigated the catalytic properties of the M468Q and T1D variants under multiple turnover conditions in solution. Fully oxidized enzymes were mixed with ABTS solution in airsaturated buffer, and multiple turnovers of oxygen consumption were monitored by using a Clark electrode (Figure S2). Michaelis-Menten parameters, $\mathrm{k}_{\mathrm{cat}}$ and $\mathrm{K}_{\mathrm{M}}$, determined for WT MoBOD were $197 \pm 5 \mathrm{~s}^{-1}$ and $551 \pm 33 \mu \mathrm{M}$, respectively, and are close to the values reported earlier for WT MoBOD. ${ }^{33}$ The M468Q variant showed low activity towards ABTS (Figure S2B). The formal reduction potential of $\mathrm{ABTS}\left(\mathrm{E}^{0}\left(\mathrm{ABTS}^{\bullet-} / \mathrm{ABTS}^{2-}\right)=680 \mathrm{mV} \text { vs. } \mathrm{NHE}\right)^{49}$ is close to the reduction potential of the $\mathrm{T} 1$ site in $\mathrm{WT}\left(570 \mathrm{mV}\right.$ vs. NHE) ${ }^{24}$ but it is significantly higher than the redox potential of the T1 site in the M468Q mutant determined to be $364 \mathrm{mV}$ vs. NHE (Figure S3). The redox potential of the T1 site is lower in the M468Q mutant since Gln is a better donor ligand than Met, thus stabilizing the oxidized more than the reduced site. Thus, the reduced activity of the M468Q mutant towards ABTS can be explained by a lowering of the redox potential of the T1 
site, which reduces the driving force for electron transfer from the substrate, making it the ratelimiting step in the catalytic site. With a low potential electron donor, $\mathrm{Fe}(\mathrm{CN}) 6^{4-}\left(\mathrm{E}^{0}-\mathrm{Fe}(\mathrm{CN}) 6^{3-}\right.$ $/ \mathrm{Fe}(\mathrm{CN})_{6}{ }^{4-}=425 \mathrm{mV}$ vs. NHE), the M-to-Q mutant of homologous $M$. verrucaria $\mathrm{BOD}$ has been shown to exhibit a higher turnover frequency than the WT enzyme. ${ }^{25}$ The T1D variant did not demonstrate any detectable catalytic activity (Figure S2C).

The single turnover $\mathrm{O}_{2}$ reactivity of the M468Q mutant was characterized by following the reaction of the fully reduced enzyme with air-saturated buffer by stopped-flow absorption spectroscopy (Figure 2). An intermediate with apparent maxima at 315, 380, 450, and $610 \mathrm{~nm}$ is observed. The intermediate resembles the native intermediate (NI) previously reported in Rhus vernicifera $(R v L)$ and Trametes versicvolor laccases $(T v L)$, possessing a characteristic charge transfer $(\mathrm{CT})$ band with a maximum at $365(R v L)-380(T \nu L) \mathrm{nm}$ due to the $\mu_{3}$-oxo to $\mathrm{Cu}(\mathrm{II})$ charge transfer at the TNC and a CT transition at $614 \mathrm{~nm}$ due to the oxidized $\mathrm{T} 1 \mathrm{Cu}$ site. ${ }^{9,11}$ The rapid formation of NI is followed by its slow decay to the RO form and can be monitored by the changes in absorption intensity at $380 \mathrm{~nm}$. The rate of NI formation of $1.61( \pm 0.04) \times 10^{6} \mathrm{M}^{-1} \mathrm{~s}^{-1}$ is comparable with the rates reported for $R v L$ and $T v L .^{9,11}$ The rate of NI decay $0.48( \pm 0.01) \mathrm{s}^{-1}$ is faster than the rates for $R v L$ and $T \nu L$, but paralleling the behavior in these MCOs, this NI decays much slower than the turnover frequency.

Similarly, the reaction of the fully reduced T1D mutant with air-saturated buffer was monitored by stopped-flow absorption spectroscopy (Figure 3). The reaction of the T1D with $\mathrm{O}_{2}$ leads to formation of an intermediate with apparent absorption features at 340 and $470 \mathrm{~nm}$, consistent with a peroxide intermediate (PI), as observed in T1Hg $R v L^{8,14}$ and T1D Fet3p, ${ }^{40,50}$ in which $\mathrm{O}_{2}$ is reduced to a peroxide, that bridges between the three $\mathrm{Cu}$ 's in the TNC with one $\mathrm{T} 3 \mathrm{Cu}$ still reduced. The rates of PI formation and decay were monitored by changes in absorption 
features at 340 and $470 \mathrm{~nm}$ (Figure 3B) and were determined to be $1.54( \pm 0.05) \times 10^{6} \mathrm{M}^{-1} \mathrm{~s}^{-1}$ and $0.0082( \pm 0.0003) \mathrm{s}^{-1}$, respectively. These rates are similar to values reported for $R v L .^{8,14}$

We have shown earlier that the RO form is not catalytically relevant for high potential multicopper oxidases. ${ }^{11}$ To check whether the T1D mutant can be catalytically active under multiple turnover conditions once in the catalytically relevant fully reduced state, we performed the additional measurements shown in Figure S4. The fully reduced T1D protein was added to an air-saturated solution of ABTS, and oxygen consumption was monitored by the Clark-electrode. A fast decrease of oxygen concentration corresponding to one turnover was observed, consistent with stopped-flow measurements. However, no further decrease in oxygen concentration was detected, precluding multiple turnovers.

\section{Electrocatalysis}

The electrochemical behaviors of WT and M468Q MoBOD and T1D BpBOD were investigated. Fully oxidized enzymes were adsorbed on spectrographic graphite electrodes modified with carbon-nanofibers (CNFs-SPGE), and the catalytic activity in the oxygen reduction reaction was examined in Mcllvaine ( $\mathrm{pH} 5.0)$ buffer at $23{ }^{\circ} \mathrm{C}$ by cyclic voltammetry (CV). No catalytic currents were observed under deaerated conditions for all BOD samples (Figure 4 A-C, black curves). In oxygen-saturated buffer, cathodic waves are apparent for WT and M468Q MoBOD (blue curves), indicating that the M468Q mutation does not hamper direct electron transfer between the enzyme and the electrode.

For WT MoBOD in the RO state, the onset for dioxygen reduction is observed at $800 \mathrm{mV}$ vs. NHE (Figure 4A, blue) in agreement with previously reported data. ${ }^{22,51}$ The catalytic wave for M468Q in the RO state (Figure $4 \mathrm{~b}$, blue) is shifted by $\sim 190 \mathrm{mV}$ to more cathodic potentials. The shift of the oxygen reduction onset potential to $\sim 610 \mathrm{mV}$ vs. NHE is consistent with the change of 
the T1 formal reduction potential in M468Q compared to WT MoBOD in solution as determined by the potentiometric titration (Figure S3). The absolute values of the onset potentials at which the catalytic signals are observed are somewhat higher for both WT and M468Q MoBOD than the redox potential of the T1 sites determined by titrations in solution (Electrode: 800 and $610 \mathrm{mV}$ vs. Solution: $570{ }^{24}$ and $360 \mathrm{mV}$ (NHE), respectively). This behavior is expected for physisorbed MCOs, as binding of the protein molecule results in exclusion of water at the electrode surface and thus an increase of the redox potential due to the decreased dielectric constant. ${ }^{52,53}$ Moreover the value of the onset potential may differ from the redox potential of the active site determined in the absence of catalysis due to "kinetic shifts",54 i.e. effects of the rates of the subsequent intramolecular electron transfer and oxygen reduction at the TNC site on the catalytic current. However, since the catalytic rates of WT and M468Q variants are within the same order of magnitude the shift in the onset potential can be mainly attributed to the change of the redox potential of the T1 site. Importantly, the cathodic shift in the catalytic onset with the decrease in the redox potential of the $\mathrm{T} 1 \mathrm{Cu}$ in the variant indicates that the $\mathrm{T} 1$ is the primary electron acceptor site from the electrode for both WT and M468Q MoBOD in their resting oxidized forms.

After incubation of the CNFs-SPGE modified with fully oxidized enzymes in $100 \mathrm{mM} \mathrm{Cl}^{-}$ at open circuit potential, the onset for dioxygen reduction in the first cycle is shifted cathodically for both WT and M468Q and observed at $\sim 460 \mathrm{mV}$ vs. NHE (Figure 4A and B, red curves vs. blue curves for RO). For the second consecutive $\mathrm{CV}$ scans, the onset potentials were restored to the values observed for WT and M468Q enzymes in the RO state (Figure S5). A shift of the onset potential upon addition of $\mathrm{Cl}^{-}$reflects the formation of the two-electron reduced $\mathrm{AR} \mathrm{TNC}$ form in both WT and M468Q MoBOD. This will be converted to the NI form by the full reduction of the TNC at a reductive potential of $<460 \mathrm{mV}$ vs. NHE and then the reaction with $\mathrm{O}_{2}$ results in restored 
RO behavior. ${ }^{30}$ Importantly, the shift in the onset potential with change in the state of the TNC (RO to AR) suggests the possibility of DET to the TNC. To confirm that the shift of the onset potential is due to the DET to the TNC and not due to slow reduction of the low-potential twoelectron reduced TNC via the T1 site we performed potential chrono-amperometry measurements (Figure S6). If the observed onset potential is due to slow reduction of the TNC in the AR form through the T1 site, at any potential more cathodic than the potential of the T1 site reduction of the AR TNC by intramolecular electron transfer from the T1 site will occur resulting in an increase of the catalytic current with time. As can be seen from Figure S6 for both WT and M468Q variants at applied potentials more cathodic than the potential of the $\mathrm{T} 1$ site, but more anodic than the potential of the AR TNC (710 mV vs. NHE for WT and $610 \mathrm{mV}$ vs. NHE for M468Q) no catalytic currents are observed during $300 \mathrm{~s}$ of measurements for proteins in their AR forms (red curves). Alternatively these applied potentials for proteins in their RO forms, catalytic currents due to electron transfer through the T1 sites are evident (blue curves). The results of this experiment confirm that for WT and M468Q variants in their AR forms, ET occurs directly to the TNC site.

T1D-modified CNFs-SPGE did not show any catalytic currents in oxygen-saturated buffer both with the protein in the RO state and after incubation of the protein film in $100 \mathrm{mM} \mathrm{Cl}^{-}$to generate AR (Figure 4C). Thus, the T1D variant does not demonstrate catalytic ORR due to DET to the TNC.

Both $\mathrm{Cl}^{-}$and $\mathrm{F}^{-}$are known inhibitors of MCOs; however, their mechanisms of inhibition are different. While additions of $\mathrm{Cl}^{-}$to the $\mathrm{RO}$ form of the enzyme result in the inactive twoelectron reduced $\mathrm{AR} \mathrm{TNC},{ }^{22,30,43} \mathrm{~F}^{-}$acts as a noncompetitive inhibitor by binding to the $\mathrm{T} 2 \mathrm{Cu}$ in the center of the fully oxidized TNC. ${ }^{18,42,55}$ Thus, we also investigated the effect of $\mathrm{F}^{-}$additions on the onset potential. Figure 5 shows CVs of WT (Figure 5A) and M468Q (Figure 5B) MoBOD 
before (blue) and after (green) additions of $10 \mathrm{mM} \mathrm{F}^{-}$to the RO form of the enzymes. The catalytic onset potential is shifted in the cathodic direction relative to the $\mathrm{T} 1$ potential for both enzymes (by $\sim 90 \mathrm{mV}$ and $50 \mathrm{mV}$ for WT and M468Q, respectively), while the catalytic activity as manifested in the values of the catalytic currents is not significantly affected.

Overall, lowering of the T1 potential in the M468Q variant relative to the WT shifts the onset catalytic potential in the cathodic direction, indicating that the $\mathrm{T} 1$ site is the primary electron acceptor site for proteins in the RO state. Moreover, the lack of the catalytic current in the T1D and $\mathrm{Cl}^{-}$treated T1D variant implies no catalytically relevant DET to the TNC in the RO form. The conversion to the AR in WT and M468Q variants results in a low onset potential at $\sim 460 \mathrm{mV}$ vs. NHE for both enzymes suggesting DET to the TNC in this state. However, $\mathrm{F}^{-}$binding to the TNC also lowers the onset potential in WT and M468Q in the RO state. These results are analyzed below.

\section{Analysis: Protein-Film Electrochemistry Data Modeling}

The CVs for WT and M468Q MoBOD in the RO and AR (AR' for M468Q) forms in oxygen-saturated buffer showed catalytic shapes that were not affected by stirring (Figure 4), indicating that the catalytic currents at high overpotentials are not mass transport-controlled. Under these conditions, according to the Butler-Volmer model, the rate of interfacial electron transfer increases exponentially with the overpotential, and at high enough overpotentials will exceed the rate of substrate conversion. ${ }^{56,57}$ Assuming that the rate of substrate conversion is independent of potential, the catalytic current is expected to increase at moderate driving force, where interfacial electron transfer controls the overall process, and level off at limiting values, where the enzyme kinetics controls the reaction. 
To model the electrochemical data for WT and M468Q mutants in their RO forms, we build a kinetic model based on the established catalytic mechanism for high-potential MCOs ${ }^{11}$ and using an approach similar to that of Gray and co-workers. ${ }^{52}$ This model, given in Scheme 1, assumes that the $\mathrm{T} 1 \mathrm{Cu}$ is reduced by DET from the electrode. The peroxy Intermediate (PI) was omitted from the kinetic scheme (in the conversion of $\mathrm{R}$ to $\mathrm{T} 1_{\mathrm{ox}} \mathrm{NI}$, Scheme 1 ) since the decay of PI is very fast, and the surface concentration of PI during catalytic cycling could be neglected $([\mathrm{PI}] \approx 0)$. The overall rate law for catalytic dioxygen reduction at high concentrations of oxygen can be written as a pseudo-first-order reaction:

$r=k_{c}[R]$

where $k_{c}$ is the rate of reaction with dioxygen and $[R]$ is the concentration of the fully reduced form of the enzyme. Solution of a system of differential equations for the changing concentrations of all the intermediate forms for [R] (Supporting Note 1) permits the cathodic current to be expressed as:

$i=n F r=\frac{n F \Gamma k_{f T 1} k_{T 1-T N C} k_{c} k_{i}}{k_{i} k_{T 1-T N C}\left(k_{f T 1}+k_{i}\right)+\left(k_{c} k_{T 1-T N C}+k_{c} k_{T N C-T 1}\right)\left(k_{b T 1}+k_{f T 1}\right)}$

In eq. (2), $k_{f T l}$ and $k_{b T l}$ are the forward and backward rate constants for interfacial electron transfer in Scheme 1, which can be described by the Butler-Volmer (BV) model by substituting the exchange rate term for the Marcus equation when $\Delta \mathrm{G}^{0}=0:{ }^{52,56}$

$$
\begin{aligned}
& k_{f T 1}=\frac{2 \pi}{\hbar} H(T 1)_{D A}^{2} \frac{1}{\sqrt{4 \pi \lambda_{T 1} k_{B} T}} \times \exp \left(\frac{-\lambda_{T 1}}{4 k_{B} T}\right) \exp \left[-\alpha(F / R T)\left(E-E_{T 1}{ }^{0^{\prime}}\right)\right] \\
& k_{b T 1}=\frac{2 \pi}{\hbar} H(T 1)_{D A}^{2} \frac{1}{\sqrt{4 \pi \lambda_{T 1} k_{B} T}} \times \exp \left(\frac{-\lambda_{T 1}}{4 k_{B} T}\right) \exp \left[(1-\alpha)(F / R T)\left(E-E_{T 1} 0^{\prime}\right)\right] .
\end{aligned}
$$


$k_{T 1-T N C}$ and $k_{T N C-T 1}$ are the forward and back intramolecular electron transfer constants for electron transfer between the T1 site and the TNC in the RO form (Scheme 1) and can be expressed using Marcus theory:

$k_{T 1-T N C}=\frac{2 \pi}{\hbar} H_{(T 1-T N C)}^{2} \frac{1}{D A} \frac{1}{\sqrt{4 \pi\left(\lambda_{T 1}+\lambda_{T N C}\right) k_{B} T}} \times \exp \left(-\frac{\left(\left(\lambda_{T 1}+\lambda_{T N C}\right)+e \times\left(E_{T N C}{ }^{0^{\prime}}-E_{T 1}{ }^{0^{\prime}}\right)\right)^{2}}{4\left(\lambda_{T 1}+\lambda_{T N C}\right) k_{B} T}\right)$

$k_{T N C-T 1}=\frac{2 \pi}{\hbar} H_{(T 1-T N C)_{D A}}^{2} \frac{1}{\sqrt{4 \pi\left(\lambda_{T 1}+\lambda_{T N C}\right) k_{B} T}} \times \exp \left(-\frac{\left(\left(\lambda_{T 1}+\lambda_{T N C}\right)+e \times\left(E_{T 1} 0^{\prime}-E_{T N C}{ }^{0^{\prime}}\right)\right)^{2}}{4\left(\lambda_{T 1}+\lambda_{T N C}\right) k_{B} T}\right)$

$k_{i}$ in eq. (2) is the limiting intermolecular electron transfer constant for the complete reduction of the oxidized TNC in the native intermediate $(\mathrm{NI}) ; n$ is the number of electrons transferred in the catalytic cycle (4); $\Gamma$ is the total surface concentration of the enzyme expressed in mol; $H(T 1)_{D A}$ and $H_{(T 1-T N C)_{D A}}$ are the electronic couplings between the T1 site and electrode and the T1 site and TNC, respectively; $\lambda_{T 1}$ and $\lambda_{T N C}$ are the reorganization energies of the T1 and TNC sites, respectively; $E_{T 1}{ }^{\prime}{ }^{\prime}$ and $E_{T N C}{ }^{0^{\prime}}$ are the formal redox potentials of the T1 site and TNC; $\alpha$ is the electron transfer coefficient in the BV model, $F$ is the Faraday constant, $N_{A}$ is the Avogadro constant, $\hbar$ is the Plank constant, $k_{B}$ is the Boltzmann constant, $e$ is the elementary charge, $R$ is the gas constant, and $T$ is the temperature.

Some values used as modeling parameters were taken from data available in the literature. The reorganization energy for the T1 site and electron transfer coefficient for the WT enzyme were taken from studies of a high potential MCO on carbon electrodes: $\lambda_{T 1}=0.4 \mathrm{eV}$ and $\alpha(\mathrm{WT})=0.48 .{ }^{52}$ The reorganization energy of the RO TNC of $1.5 \mathrm{eV}$ was used. ${ }^{15}$ The $k_{c}$ value for the reaction between the reduced TNC and dioxygen (Scheme 1) based on our previous studies is $4 \times 10^{3} \mathrm{~s}^{-1}$ under dioxygen saturation. ${ }^{11}$ The electronic coupling between the T1 site and TNC through the Cys-His superexchange pathway was estimated to be $H_{(T 1-T N C)} \sim \mathrm{cm}^{-1} .{ }^{13}$ Using 
these values as an input, we fit the experimental linear sweep voltammograms (LSVs) of WT and M468Q (Figure 6A) in their RO forms to eq. (2) by a non-linear least-squares procedure using the Levenberg-Marquardt algorithm. The fitting parameters are given in Table 3. As can be seen from Figure 6A, this model closely reproduces the experimental data. Importantly, this model assumes reduction through the T1 site and the shift in onset potential in going to M468Q RO in Figure 6 just reflects the decrease in the redox potential of the $\mathrm{T} 1$ site by $\sim 180 \mathrm{mV}$.

The experimental LSVs for $\mathrm{F}^{-}$inhibition of WT and M468Q could also be reproduced using Scheme 1 by allowing for only a minor variance in the fitting parameters within the fitting errors (Table 3) and decreasing the $E_{T N C}{ }^{0^{\prime}}$ by $\sim 150 \mathrm{mV}$ (i.e. the reduction potential decrease due to $\mathrm{F}^{-}$ binding to the fully oxidized TNC). ${ }^{19,20}$ Here reduction is still through the T1 site (fit to eq. (2) included in magenta dashed lines, Figure $6 \mathrm{C}, \mathrm{D})$ and the shift to more cathodic potentials is due to the larger overpotential required for the rate of electron transfer from the electrode to the T1 site $\left(k_{f T l}\right.$, Scheme 1) to exceed the back-electron transfer rate from the fluoride-bound-lower potential TNC $\left(k_{T N C-T l}\right.$, Scheme 1).

Alternately, we could not fit experimental data for WT and M468Q treated with $\mathrm{Cl}^{-}$, resulting in the AR form of the TNC, with reasonable parameters using the kinetic Scheme 1 . Imputing the lower reorganization energy of the $\mathrm{AR}$ form $(0.75 \mathrm{eV}$ relative to the $\mathrm{RO}$ value of 1.5 eV) ${ }^{43}$ into eqs. (5) and (6), letting the potential of the AR TNC site float and fixing all other fitting parameters (Table 3) results in the poor fits shown in Figure S7A (the adjusted $\mathrm{R}^{2}$ are 0.251 (WT) and 0.916 (M468Q) vs. 0.999 for both WT and M468Q in their RO forms). Even allowing for a minor variance $(10 \%)$ in all the fitting parameters in Table 3, the fits in Figure S7A cannot be improved. These Scheme 1 fits to the AR LSV can be improved if we permit all the parameters to float within the broad range specified in Table S1 (Figure S7B), however, they still do not 
reproduce the shape of the experimental LSVs (the adjusted $\mathrm{R}^{2}$ are 0.962 (WT) and 0.983 (M468Q)). To satisfactory fit, the experimental LSVs of WT and M468Q in their alternative resting forms using Scheme 1 the reduction potentials of the T1 sites must be allowed to decrease by $\sim 400 \mathrm{mV}$ for $\mathrm{WT}$ and $\sim 250 \mathrm{mV}$ for M468Q, which is not physically reasonable as perturbations of the TNC site do not change the $\mathrm{T} 1$ site potential by more than $50 \mathrm{mV} .{ }^{58}$

Based on experimental observations, it was suggested earlier that the reactivation of the chloride-treated protein (i.e. the two-electron reduced AR form of the TNC) occurs by electron transfer directly to the TNC site. ${ }^{30,43}$ The modified kinetic scheme accounting for the direct electron transfer to the two-electron reduced AR TNC $\left(k_{f T N C}, k_{b T N C}\right)$ is shown in Scheme 2. In this scheme, the first ET occurs directly to the AR TNC site forming the fully reduced (R) protein, which reacts with $\mathrm{O}_{2}$ to generate NI. On subsequent turnovers, electron transfer to the NI TNC occurs through the T1 site. The cathodic current for this Scheme 2 (Supporting Note 2) can be expressed as follows:

$$
i=\frac{n F \Gamma k_{f T N C} k_{f T 1} k_{c} k_{i}}{k_{i} k_{b T N C} k_{f T 1}+k_{i} k_{f T N C} k_{f} T 1+k_{c} k_{f T N C} k_{f T 1}+k_{c} k_{f T N C}\left(k_{b T 1}+k_{i}\right)}
$$

Interfacial electron transfer rate constants to the T1 site, $k_{f T l}$ and $k_{b T l}$ are again given by eq. (3) and (4), respectively. $\mathrm{k}_{f T N C}$ and $k_{b T N C}$, the forward and backward rate constants for direct electron transfer to the TNC, are expressed similarly by substituting the two-electron reduced AR TNC as the primary electron acceptor site:

$$
\begin{aligned}
& k_{f T N C}=\frac{2 \pi}{\hbar} H(T N C)_{D A}^{2} \frac{1}{\sqrt{4 \pi \lambda_{T N C(A R)} k_{B} T}} \times \exp \left(\frac{-\lambda_{T N C(A R)}}{4 k_{B} T}\right) \exp \left[-\alpha(F / R T)\left(E-E_{T N C(A R)}{ }^{0^{\prime}}\right)\right] \\
& k_{b T N C}=\frac{2 \pi}{\hbar} H(T N C)_{D A}^{2} \frac{1}{\sqrt{4 \pi \lambda_{T N C(A R)} k_{B} T}} \times \exp \left(\frac{-\lambda_{T N C(A R)}}{4 k_{B} T}\right) \exp \left[(1-\alpha)(F / R T)\left(E-E_{T N C(A R)} 0^{\prime}\right)\right]
\end{aligned}
$$


Using the parameters obtained for $k_{i}, \alpha(T 1), E_{T 1}{ }^{\prime}$ and $\Gamma$ in fitting Scheme 1 to the data for RO forms of the enzymes (Table 3), we fit the additional parameters in eq. (7) describing Scheme 2 to the experimental LSVs for WT and M468Q in their AR forms. The new parameters obtained for DET to the AR TNC are summarized in Table 4. The values obtained for $H(T N C)_{D A}\left(\sim 0.001 \mathrm{~cm}^{-}\right.$

$\left.{ }^{1}\right)$ are close to the $H(T 1)_{D A}$ values and $E_{T N C(A R)}{ }^{0^{\prime}} \approx 350 \mathrm{mV}$ vs. NHE for both proteins, which is close to the redox potential of the AR form of the TNC obtained experimentally ${ }^{59}$ and estimated by DFT calculations. ${ }^{43}$ Figure 6B shows an overlay of the LSVs calculated using eq. (7) (Scheme 2) with the experimental data.

\section{DISCUSSION}

\section{Electronic Coupling between the Electrode and the T1 and TNC sites}

The mean value of the electronic couplings H(T1)DA determined from analysis of the WT and M468Q data from the RO forms $\left(\sim 0.002 \mathrm{~cm}^{-1}\right.$, Table 3) can be utilized to estimate the average distance between the T1 site and electrode surface. ${ }^{52}$ Following Gray and co-workers (Supporting Note 3) the average distance between the electrode and T1 site in both WT and M468Q mutants is estimated to be $\sim 25 \AA$. This value is close to the mean distance between the T1 site and electrode (see eq. (s25)), determined from the crystal structures of homologous $M$. verrucaria BOD (the closest distance is $7 \AA$ and the maximum distance is $49 \AA$ based on PDB:6IQZ and 6IQX structures for WT and the M-to-Q mutant, respectively). The results suggest that enzyme molecules are randomly oriented on the electrode surface, in agreement with previous reports. ${ }^{52,57}$ Importantly the observation of DET to the TNC in the AR form has enabled us to experimentally obtain the value of $\mathrm{H}(\mathrm{TNC})_{\mathrm{DA}} \sim 0.001 \mathrm{~cm}^{-1}$ (Table 4). This also gives an average distance between the TNC and the electrode of $\sim 25 \AA$, which is close to the mean distance between the TNC and electrode 
(the closest distance is $14 \AA$ and the maximum distance is $45 \AA$ based on PDB:6IQZ and 6IQX structures for the WT and the M-to-Q mutant, respectively). Thus, our findings show that the electronic coupling between the T1 site and electrode is similar to the electronic coupling between the TNC and the electrode, and there is no preferential electron transfer pathway for DET to the T1 site.

\section{Effect of the TNC reorganization energy on the electron transfer pathway}

The experimentally observed shift of the catalytic onset potential in the M468Q mutant (Figure 4A, B) and the absence of a catalytic current in the T1D mutant (Figure 4C) clearly demonstrate that electron transfer to the TNC in the RO form occurs through the T1 site.

We first consider DET to the RO TNC. Replacing the primary electron acceptor site from the $\mathrm{T} 1$ to the RO TNC in eq. (3), the rate of DET to the TNC can be calculated. The reorganization energy of the TNC in the RO form is $1.5 \mathrm{eV}^{15}$ and $\mathrm{H}(\mathrm{TNC})_{\mathrm{DA}}$ obtained from LSV data fitting is $0.001 \mathrm{~cm}^{-1}$ (Table 3). At zero overpotential (the applied potential is equal to the potential of the TNC), the DET rate to the TNC ( $k_{f T N C}$, Figure 7$)$ is calculated to be $\approx 10^{-4} \mathrm{~s}^{-1}$, and even at high applied overpotentials $\left(E_{T N C}{ }^{0^{\prime}}-E_{\text {applied }} \approx 300 \mathrm{mV}\right)$ the rate of electron transfer to the TNC ( $k_{f T N C}$, Figure 7) will not exceed $0.02 \mathrm{~s}^{-1}$. Using the electrode coverage determined by analysis of the LSV data ( $\Gamma$ in Table 3) and the electron transfer rate of $0.02 \mathrm{~s}^{-1}$, the estimated current of DET to the TNC should be on the order of $2 \times 10^{-9} \mathrm{~A}$, which is lower than the limit of detection in our experimental setup $\left(>10^{-7} \mathrm{~A}\right)$. This estimation is consistent with the experimentally observed absence of a detectable catalytic current in the T1D mutant (Figure 4C).

We further consider the possibility of DET to the TNC in the catalytically relevant NI form generated after the first turnover. The reorganization energy of the TNC in the NI was estimated 
to be $1.2 \mathrm{eV} .{ }^{15}$ While this reorganization energy is lower than that of RO, it still gives a very low electron transfer rate for DET to NI $\left(0.7 \mathrm{~s}^{-1}\right.$ at $\sim 300 \mathrm{mV}$ overpotential $)$ not consistent with the high catalytic currents experimentally observed under multiple turnover conditions $\left(\sim 1.75 \times 10^{-5} \mathrm{~A}\right.$ experimental for WT in Figure 4A, blue curve vs. $7 \times 10^{-8}$ A calculated for NI using the rate of $\left.0.7 \mathrm{~s}^{-1}\right)$

Alternately, the electron transfer rate to the TNC through the T1 site can be estimated considering two consecutive steps (Figure 7):

Step 1. Interfacial electron transfer between the electrode and the T1 site ( $k_{f T l}$ in Figure 7), with the $\mathrm{T} 1$ site reorganization energy of $0.4 \mathrm{eV}$ and $\mathrm{H}(\mathrm{T} 1)_{\mathrm{DA}} \sim 0.002 \mathrm{~cm}^{-1}$ (Table 3). At zero overpotential this gives a rate of $\sim 10 \mathrm{~s}^{-1}$.

Step 2. The intramolecular electron transfer between the T1 site and the TNC $\left(k_{T 1-T N C}\right.$ for forward electron transfer and $k_{T N C-T l}$ for back electron transfer in Figure 7). Depending on the state of the TNC (RO, NI or AR) the forward and back intramolecular electron transfer rates will differ due to the difference in the reorganization energies and for the AR TNC additionally due to the lower reduction potential of the TNC site:

a. For the RO TNC (first turnover) the reorganization energy $\lambda=\lambda_{T 1}+\lambda_{T N C(R O)}=1.9$ $\mathrm{eV}$, the $\Delta E^{0^{\prime}}$ is $\sim 40 \mathrm{mV}$ for $\mathrm{WT}$ and $240 \mathrm{mV}$ for M468Q (Table 3), and the electronic coupling through the Cys-His superexchange pathway of $H_{(T 1-T N C)} \sim 1 \mathrm{~cm}^{-1} \cdot{ }^{13}$ Using eqs. (5) and (6), this gives the forward rates $\left(k_{T 1-T N C}\right)$ of $15 \mathrm{~s}^{-1}$ (WT) and $120 \mathrm{~s}^{-1}$ (M468Q), while the back electron transfer rates $\left(k_{T N C-T I}\right)$ are $0.7 \mathrm{~s}^{-1}(\mathrm{WT})$ and $0.01 \mathrm{~s}^{-1}$ (M468Q). 
b. For the NI TNC (after the first turnover) $\lambda=\lambda_{T 1}+\lambda_{T N C(N I)}=1.6 \mathrm{eV}$, the $\Delta E^{0^{\prime}}$ is $\sim 40 \mathrm{mV}$ for WT and $240 \mathrm{mV}$ for M468Q.This gives the forward rates of $280 \mathrm{~s}^{-1}$ (WT) and $370 \mathrm{~s}^{-1}$ (M468Q) consistent with the observed rates of the intramolecular electron transfer to NI of $k_{i} \sim 240$ and $310 \mathrm{~s}^{-1}$ for WT and M468Q, respectively (Scheme 1 and Table 3), obtained by fitting the experimental data to Scheme 1 . The back electron transfer rates are $11 \mathrm{~s}^{-1}(\mathrm{WT})$ and $0.1 \mathrm{~s}^{-1}(\mathrm{M} 468 \mathrm{Q})$.

c. For the AR TNC the reorganization energy $\lambda=\lambda_{T 1}+\lambda_{T N C(A R)}=1.15 \mathrm{eV},{ }^{43} \Delta E^{0^{\prime}}$ is $-430 \mathrm{mV}$ for WT and $-210 \mathrm{mV}$ for M468Q (Table 3, and for $E^{0^{\prime}}$ (TNC AR) Table 4). From eqs. (5) and (6), the forward rates $\left(k_{T I-T N C}\right)$ are $~ 0.2 \mathrm{~s}^{-1}$ (WT) and $35 \mathrm{~s}^{-1}$ (M468Q), while the back electron transfer rates $\left(k_{T N C-T 1}\right)$ are $2 \times 10^{6} \mathrm{~s}^{-1}$ and $10^{5} \mathrm{~s}^{-1}$ for WT and M468Q, respectively.

Thus, at zero overpotential for both the WT and the M468Q variant in their RO forms, the electron transfer rate to the TNC through the T1 site is $\sim 10 \mathrm{~s}^{-1}$ and is controlled by the interfacial electron transfer (Step 1). So electron transfer to the TNC in the RO form via the T1 site is $\sim 10^{5}$ times faster than direct electron transfer to the TNC from the electrode. This is due to the much higher reorganization energy of the RO TNC compared to the $\mathrm{T} 1$ site, which in the case of electron transfer through the T1 site is compensated by the strong electronic coupling through the Cys-His superexchange pathway. For this T1 to TNC electron transfer, the reorganization energy of the TNC is included in Step 2a, for which the electronic coupling $\left(H_{(T 1-T N C)} \sim 1 \mathrm{~cm}^{-1}\right)$ is $10^{3}$ times stronger than the electronic coupling between the electrode and the TNC $\left(\mathrm{H}(\mathrm{TNC})_{\mathrm{DA}} \sim 0.001 \mathrm{~cm}^{-1}\right)$. This results in a significantly higher $\mathrm{T} 1$ to TNC electron transfer rate due to quadratic dependence of the ET rate on $\mathrm{H}_{\mathrm{DA}}$ according to Marcus theory. ${ }^{60}$ 
At high overpotentials, electron transfer to the TNC through the T1 site is controlled by the intramolecular electron transfer to the NI for both WT and M468Q proteins (Step 2b) and is also much larger ( 240 $\mathrm{s}^{-1}$ (WT) and $310 \mathrm{~s}^{-1}$ (M468Q)) compared to the calculated DET rate to the TNC in the NI form $\left(0.7 \mathrm{~s}^{-1}\right.$, vide supra $)$ Thus, while there is little difference in the T1/electrode and TNC/electrode electronic couplings, electron transfer to the TNC in the RO and NI forms occurs primarily via the $\mathrm{T} 1$ site due to the high reorganization energy of the fully oxidized RO and NI forms of the TNC because of the efficient electronic coupling between the T1 and TNC.

Due to the lower reorganization of the AR TNC and its lower reduction potential, DET to the AR TNC can be observed experimentally (Figure 4A, B red curves). In the case of electron transfer to the AR TNC through the T1 site, the forward intramolecular electron transfer $\left(k_{T 1-T N C}\right.$, Step 2c) is uphill, while the back electron transfer from the TNC to the T1 site $\left(k_{T N C-T I}\right)$ is thermodynamically favorable. This is opposite to the T1 to the TNC electron transfer in the RO and NI forms, where the electron transfer from the T1 site to the TNC is downhill. For both WT and M468Q in their AR forms, $k_{T N C-T 1}$ are significantly faster than the reaction rate of the fully reduced TNC with oxygen $\left(k_{c} \approx 4 \times 10^{3} \mathrm{~s}^{-1}\right.$, Figure 7$)$. Thus, the TNC in the AR form can not be reduced through the T1 site and can not react with oxygen until $k_{f T l}$ exceeds $k_{T N C \text {-Tl }}$ to prevent the fast back electron transfer. $k_{f T l}$ will exceed $k_{T N C-T l}$ at applied potentials of $\sim 120$ and $60 \mathrm{mV}$ vs. NHE for WT and M468Q, respectively. However, the catalytic currents due to the ORR are detectable already at $\sim 460 \mathrm{mV}$ vs. NHE for both enzymes (Figure 4, red curves and Figure 6B). This experimental observation rules out electron transfer to the AR TNC via the T1 site. Using the reorganization energy of the AR TNC $0.75 \mathrm{eV}$ and $\mathrm{H}(\mathrm{TNC})_{\mathrm{DA}} \approx 0.001 \mathrm{~cm}^{-1}$ obtained from the LSV data (Table 4), the direct electron transfer rate to the AR form of the TNC ( $k_{f T N C}$, Figure 7$)$ at zero overpotential is estimated to be $0.2 \mathrm{~s}^{-1}$ which is consistent with catalytic currents recorded for the 
WT and M468Q AR forms at this potential $\left(\mathrm{E}_{\text {applied }}=350 \mathrm{mV}\right.$, Figure $6 \mathrm{~B}$ dashed cyan curves (calculated) vs. red (WT) and pink (M468Q) curves (experimental)).

Thus, the AR form is reduced by direct electron transfer from the electrode to the TNC. It is important to emphasize that once the AR form is fully reduced, it reacts with dioxygen to convert to the catalytically relevant NI form (Scheme 2). Then the electron transfer occurs through the T1 site producing the catalytic current. Moreover, as experimentally demonstrated by the contrast of the TNC perturbations by $\mathrm{Cl}^{-}$(i.e. its two-electron reduction to $\mathrm{AR}$ ) vs. $\mathrm{F}^{-}$(i.e. lowering the $E_{T N C}{ }^{\prime}$ of RO), lowering the TNC potential relative to the potential of the T1 site is not sufficient to change the primary electron acceptor site without a change in the reorganization energy of the TNC. Overall, the first electron transfer step for proteins in the AR form occurs directly to the TNC due to the lower reorganization energy of this already two-electron reduced cluster, while all subsequent interfacial electron transfer steps to NI under multiple turnover conditions proceed through the T1 site.

\section{Implications for biofuel cells design}

The possibility of MCO immobilization with controlled orientation on electrode surfaces has been discussed in several reports. ${ }^{31,61-63}$ Site-specific immobilization of high potential MCOs enabling close contact between the electrode and TNC is expected to result in a superior ORR catalyst since in this case, the turnover frequency would be controlled by the rate of dioxygen reduction by the TNC bypassing slow intramolecular electron transfer. ${ }^{31}$ Using eq. (s22) and the closest distance between the TNC and electrode of $14 \AA$ (PDB:6IQZ), the electronic coupling for such a controlled orientation is estimated to be $\sim 0.27 \mathrm{~cm}^{-1}$. For this $\mathrm{H}_{\mathrm{DA}}$, the electron transfer rate to the TNC in the NI form (i.e. after the first turnover) will exceed the rate-limiting intramolecular 
$\mathrm{T} 1$ to the NI TNC electron transfer rate in WT protein at $\sim 50 \mathrm{mV}$ overpotential. In single molecule electrochemical studies of high potential $T v L$ randomly colliding with the electrode surface, individual protein molecules were observed to have high turnover frequencies exceeding the intramolecular electron transfer rate at high applied overpotentials. ${ }^{64}$ Such proximity of the TNC to the electrode surface, however, is challenging to achieve experimentally when immobilizing protein molecules, and some distribution of orientations will inevitably occur, leading to lower electronic coupling and thus requiring higher overpotentials for DET to the TNC. Figure 8 presents the dependence of the overpotential required for the turnover frequency by DET to the NI TNC to exceed the intramolecular electron transfer rate on the average distance between the electrode and the TNC. As can be seen already at an average distance of $\sim 19 \AA$, a large overpotential $(>300 \mathrm{mV})$ is required for DET to the NI TNC to be faster than electron transfer through the T1 site.

\section{CONCLUSIONS}

We have carried out a systematic study of the electron transfer between an electrode and immobilized high potential MCOs using electrochemical methods combined with data modeling of electrode kinetics. As found earlier, the protein molecules are randomly oriented on the electrode surface. This results in little difference in the T1/electrode vs. TNC/electrode electronic couplings. While there is no preferential orientation of protein molecules to favor DET through the $\mathrm{T} 1$ site, electron transfer to the TNC in the RO and NI forms occurs primarily through the $\mathrm{T} 1$ site due to the high reorganization energy of the fully oxidized TNC and its efficient electronic coupling to the $\mathrm{T} 1 \mathrm{Cu}$. We have further determined that the lower reorganization energy of the TNC in the AR form does enable DET to the TNC, however, this only occurs in the first turnover (Figure S5). We have discussed the implications of our findings for the design of more efficient MCO-based cathodes for the ORR. 


\section{ASSOCIATED CONTENT}

Supporting information. Derivation of the cathodic current expressions for Schemes 1 and 2, the average $\mathrm{T} 1$ site/electrode distance estimation, potentiometric titration of the Type $1 \mathrm{Cu}$ site in M468Q MoBOD, additional kinetic and CV data, potential chrono-amperometry measurements, fits of the AR LSVs to Scheme 1.

\section{AUTHOR INFORMATION}

*Corresponding Authors

Edward I. Solomon, Email: solomone@stanford.edu

Alina Sekretareva, Email: alina.sekretareva@kemi.uu.se

\section{ACKNOWLEDGMENTS}

E.I.S. thanks the National Institutes of Health (DK031450) for supporting this research. A.S. acknowledges support from the Knut and Alice Wallenberg Foundation (KAW 2015.0418). 
Table 1. MoBOD and BpBOD Copper Quantitation

$\begin{array}{cccc}\text { Protein } & \mathrm{Cu}(\mathrm{I}) & \text { Paramagnetic } \mathrm{Cu} & \text { Total } \mathrm{Cu} \\ & (\text { anaerobic } & (\mathrm{EPR}) & \text { (biquinoline }\end{array}$

biquinoline assay assay)

\begin{tabular}{lccc}
\hline WT MoBOD as isolated & 0.7 & 2.1 & 3.8 \\
\hline WT MoBOD fully oxidized & 0.1 & 1.8 & 3.9 \\
\hline WT MOBOD + 100 mM NaCl & 2.0 & 1.9 & 3.9 \\
\hline M468Q MoBOD as isolated & 1.8 & 1.7 & 4.1 \\
\hline M468Q MoBOD fully oxidized & 0 & 1.7 & 4.0 \\
\hline M468Q MoBOD + 100 mM NaCl & 2.2 & 1.9 & 4.1 \\
\hline T1D BpBOD fully oxidized & - & 1.1 & 2.7 \\
\hline
\end{tabular}


Table 2. Spin-Hamiltonian Parameters.

\begin{tabular}{|c|c|c|}
\hline Cu site & $g_{x}, g_{y}, g_{z}$ & $A_{x}, A_{y}, A_{z}\left(\times 10^{-4} \mathrm{~cm}^{-1}\right)$ \\
\hline \multicolumn{3}{|c|}{ WT RO } \\
\hline $\mathrm{T} 1$ & $2.043,2.058,2.220$ & $8.5,4.2,73.1$ \\
\hline $\mathrm{T} 2$ & $2.053,2.064,2.257$ & $31.7,11.2,178.9$ \\
\hline \multicolumn{3}{|c|}{ WT AR } \\
\hline $\mathrm{T} 1$ & $2.043,2.058,2.220$ & $8.5,4.2,73.1$ \\
\hline AR & $2.037,2.106,2.370$ & $28.9,20.1,80.0$ \\
\hline \multicolumn{3}{|c|}{ M468Q RO } \\
\hline $\mathrm{T}^{\prime}{ }^{\prime}$ & $2.058,2.067,2.276$ & $40.1,11.5,41.1$ \\
\hline $\mathrm{T} 2$ & $2.053,2.064,2.257$ & $31.7,11.2,178.9$ \\
\hline \multicolumn{3}{|c|}{ M468Q AR' } \\
\hline $\mathrm{T}^{\prime}{ }^{\prime}$ & $2.058,2.067,2.276$ & $40.1,11.5,41.1$ \\
\hline $\mathrm{AR}^{\prime}$ & $2.048,2.082,2.228$ & $49.1,1.0,183.3$ \\
\hline \multicolumn{3}{|c|}{ T1D RO } \\
\hline $\mathrm{T} 2$ & $2.053,2.064,2.257$ & $31.7,11.2,178.9$ \\
\hline
\end{tabular}


Table 3. Fitting parameters obtained from modeling of the LSV data for the WT and fluoridetreated proteins using Scheme 1.

\begin{tabular}{|c|c|c|}
\hline & WT RO & M468Q RO \\
\hline $\mathrm{H}(\mathrm{T} 1)_{\mathrm{DA}}, \mathrm{cm}^{-1}$ & $0.0020 \pm 0.0005$ & $0.0016 \pm 0.0002$ \\
\hline$\alpha(\mathrm{T} 1)$ & Fixed at 0.48 & $0.32 \pm 0.05$ \\
\hline $\mathrm{k}_{\mathrm{i}}, \mathrm{s}^{-1}$ & $240 \pm 20$ & $310 \pm 30$ \\
\hline$E_{T 1}{ }^{0^{\prime}}, m V$ vs. NHE & $740 \pm 30$ & $560 \pm 30$ \\
\hline$E_{T N C}{ }^{\prime \prime}, m V v s . N H E$ & $780 \pm 50$ & $800 \pm 50$ \\
\hline$E_{T N C+F-}{ }^{0^{\prime}}, m V$ vs. $N H E$ & $640 \pm 30$ & $600 \pm 30$ \\
\hline$\Gamma, \mathrm{mol}$ & $(2.6 \pm 0.5) \times 10^{-13}$ & $(2.4 \pm 0.5) \times 10^{-13}$ \\
\hline
\end{tabular}


Table 4. Fitting parameters obtained from modeling of the LSV data for the chloride-treated AR forms of the MCOs using Scheme 2.

\begin{tabular}{lcc}
\hline & WT AR & M468Q AR \\
\hline $\mathrm{H}(\mathrm{TNC})_{\mathrm{DA}}, \mathrm{cm}^{-1}$ & 0.001 & 0.0009 \\
\hline$\alpha(\mathrm{TNC})$ & \multicolumn{2}{c}{ Not sensitive } \\
\hline $\boldsymbol{E}_{\boldsymbol{T N C}(\boldsymbol{A R})}^{\mathbf{0}^{\prime}}, \boldsymbol{m V} \boldsymbol{v}$ s. $\boldsymbol{N H E}$ & 350 & 350 \\
\hline
\end{tabular}



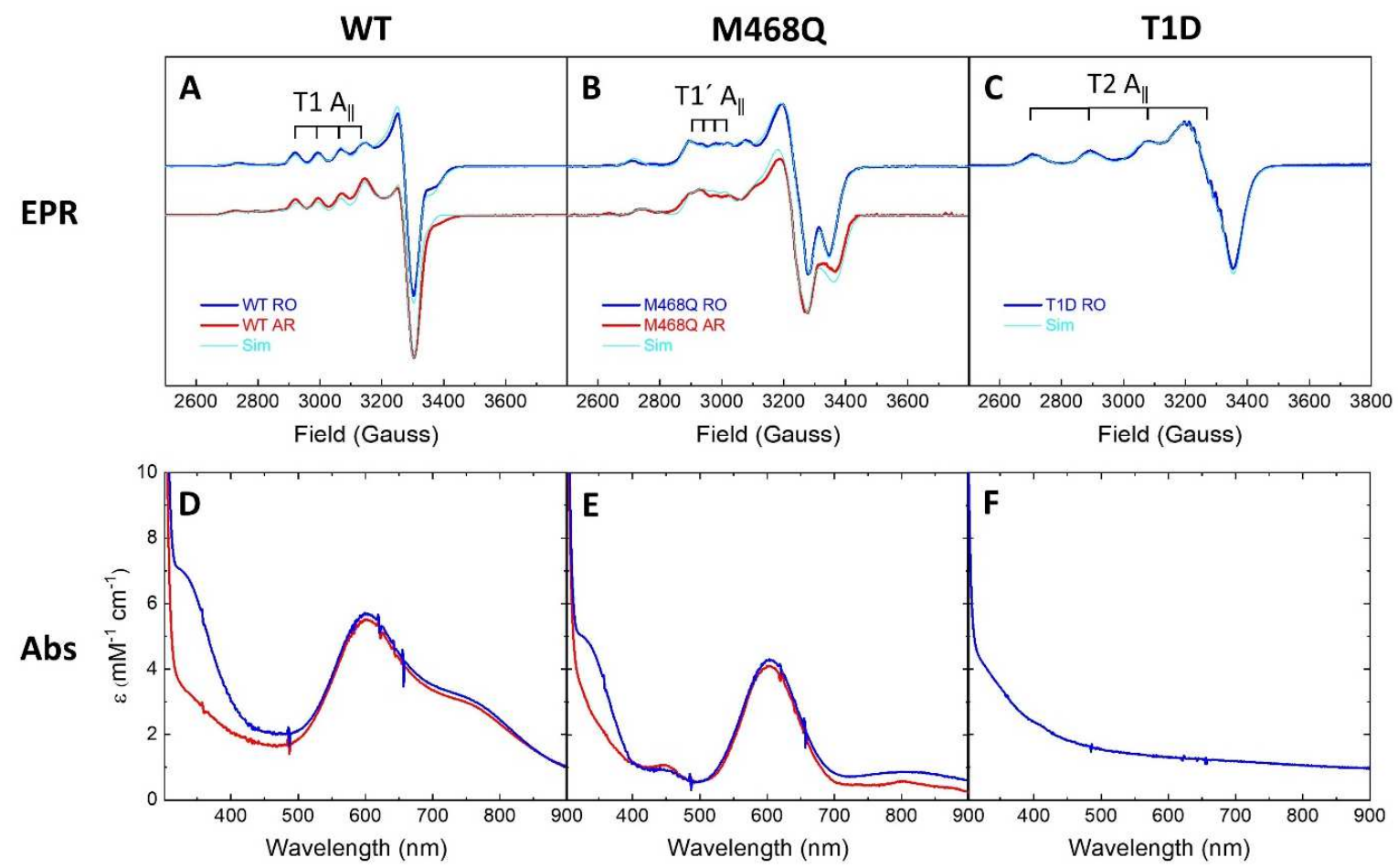

Figure 1. 77K X-band EPR spectra (A-C) and room-temperature electronic absorption spectra (DF) of the fully oxidized enzyme before (blue) and after addition of $100 \mathrm{mM} \mathrm{Cl}^{-}$(red): (A),(D) WT MoBOD, (B),(E) M468Q MoBOD and (C),(F) T1D BpBOD. EPR simulated spectra are shown in cyan. 

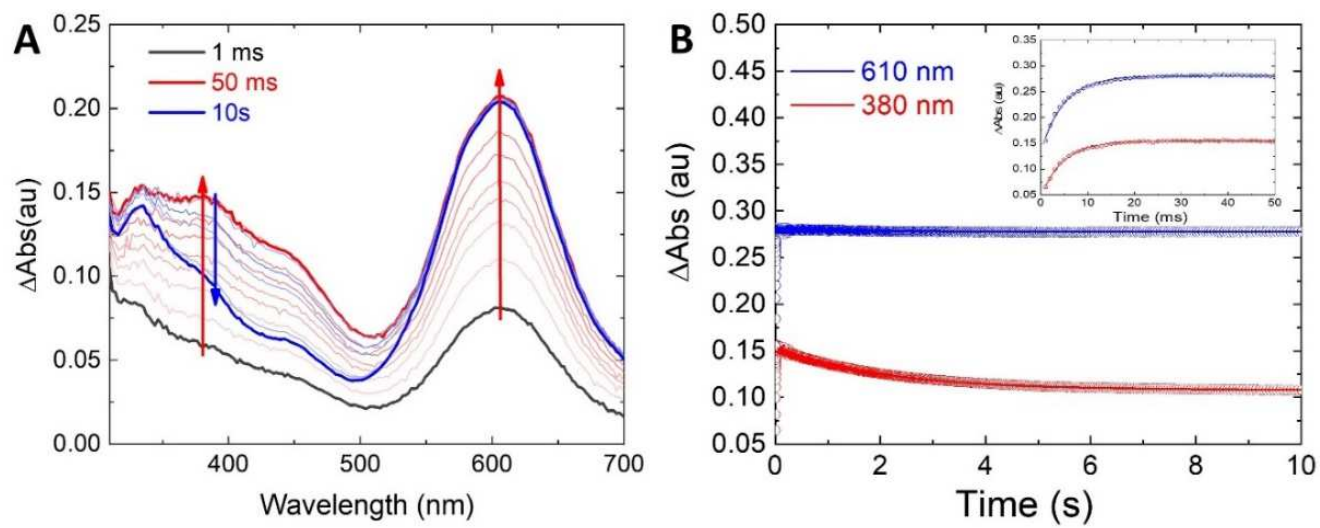

Figure 2. The reaction of the reduced M468Q mutant with $\mathrm{O}_{2}$ monitored by a rapid scan stoppedflow instrument in Mcllvaine buffer $(\mathrm{pH} 5.0)$ at $23^{\circ} \mathrm{C}$; $[\mathrm{M} 468 \mathrm{Q}]=50 \mu \mathrm{M},\left[\mathrm{O}_{2}\right]=130 \mu \mathrm{M}$. (A) Absorption spectra from $1 \mathrm{~ms}$ to $10 \mathrm{~s}$ (B) Absorption traces (circles) and fits (lines) of the T1 (610 $\mathrm{nm}$, blue) and TNC (380 nm, red) bands in the reduction of the native intermediate. 

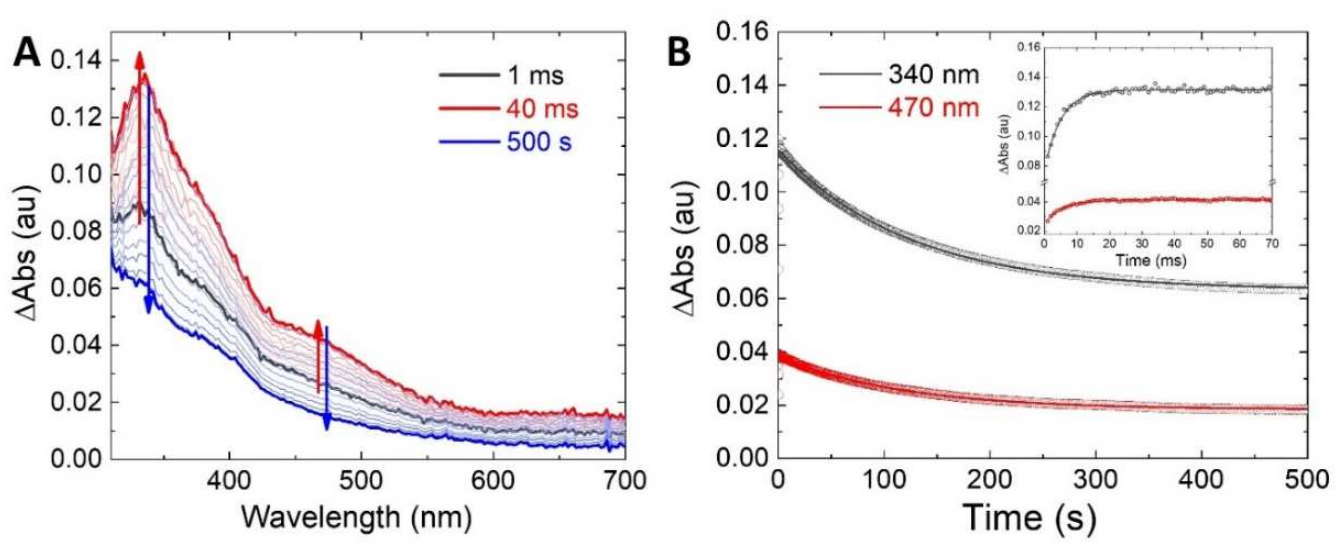

Figure 3. The reaction of the reduced T1D mutant with $\mathrm{O}_{2}$ monitored by a rapid scan stoppedflow instrument in Mcllvaine buffer $\left(\mathrm{pH}\right.$ 5.0) at $23{ }^{\circ} \mathrm{C}$; [T1D] $=35 \mu \mathrm{M},\left[\mathrm{O}_{2}\right]=130 \mu \mathrm{M}$. (A) Absorption spectra from $1 \mathrm{~ms}$ to $500 \mathrm{~s}$ (B) Absorption traces (circles), and fits (lines) of the peroxy intermediate formation (insert) and decay (340 nm, black) and (470 nm, red). 


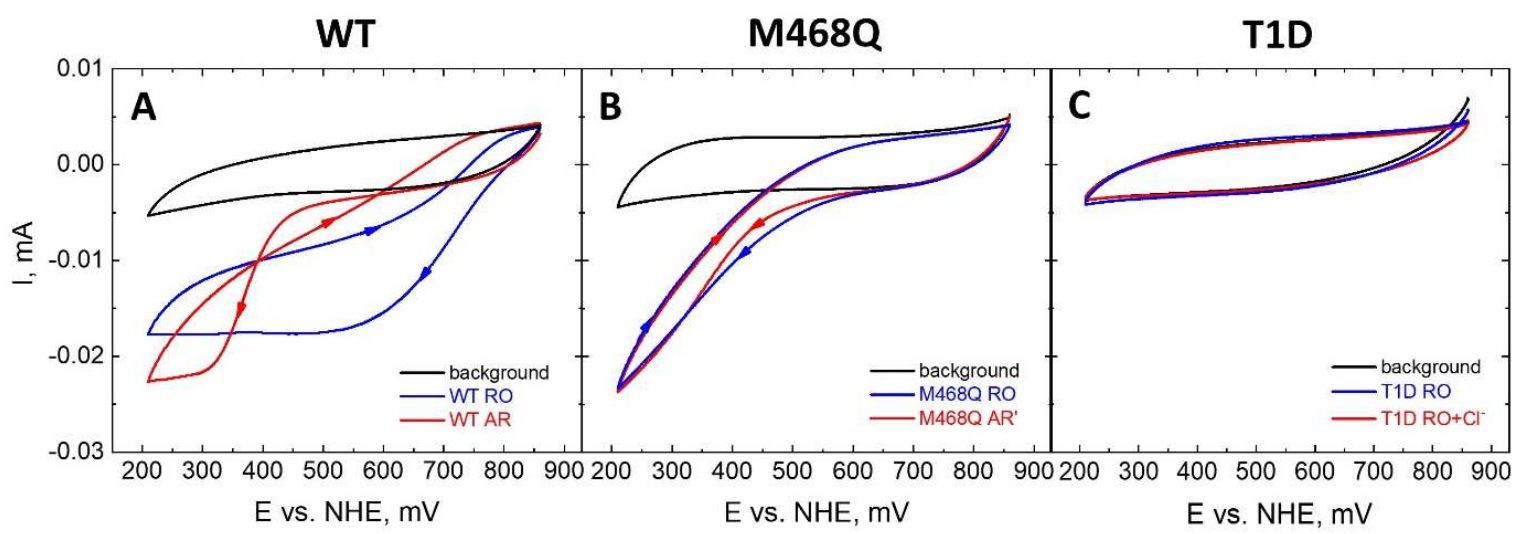

Figure 4. Cyclic voltammograms of CNFs-modified SPGEs with the immobilized enzyme in $\mathrm{N}_{2}-$ saturated buffer (black), the reoxidized enzyme before (blue) and after addition of $100 \mathrm{mM} \mathrm{Cl}^{-}$ (red) in $\mathrm{O}_{2}$-saturated buffer. (A) WT MoBOD, (B) M468Q MoBOD, and (C) T1D BpBOD. Mcllvaine buffer ( $\mathrm{pH} \mathrm{5.0)}$ at $23{ }^{\circ} \mathrm{C}$. Scan rate $2 \mathrm{mV} \mathrm{s}^{-1}$. Arrows indicate scan direction; negative current represents reduction. 


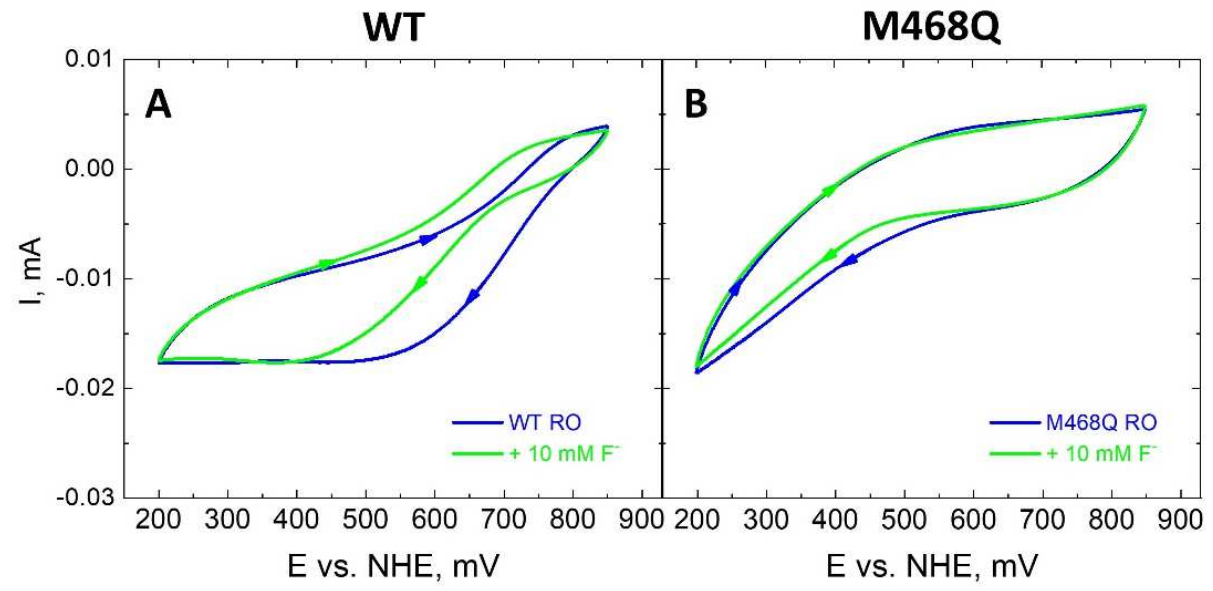

Figure 5. Cyclic voltammograms of CNFs-modified SPGEs with immobilized MoBOD in the resting oxidized form before (blue) and after addition of $10 \mathrm{mM} \mathrm{F}^{-}$(green) in $\mathrm{O}_{2}$-saturated Mcllvaine buffer (pH 5.0) at $23{ }^{\circ} \mathrm{C}$. (A) WT MoBOD, (B) M468Q MoBOD. Scan rate $2 \mathrm{mV} \mathrm{s}^{-1}$. Arrows indicate scan direction; negative current represents reduction. 

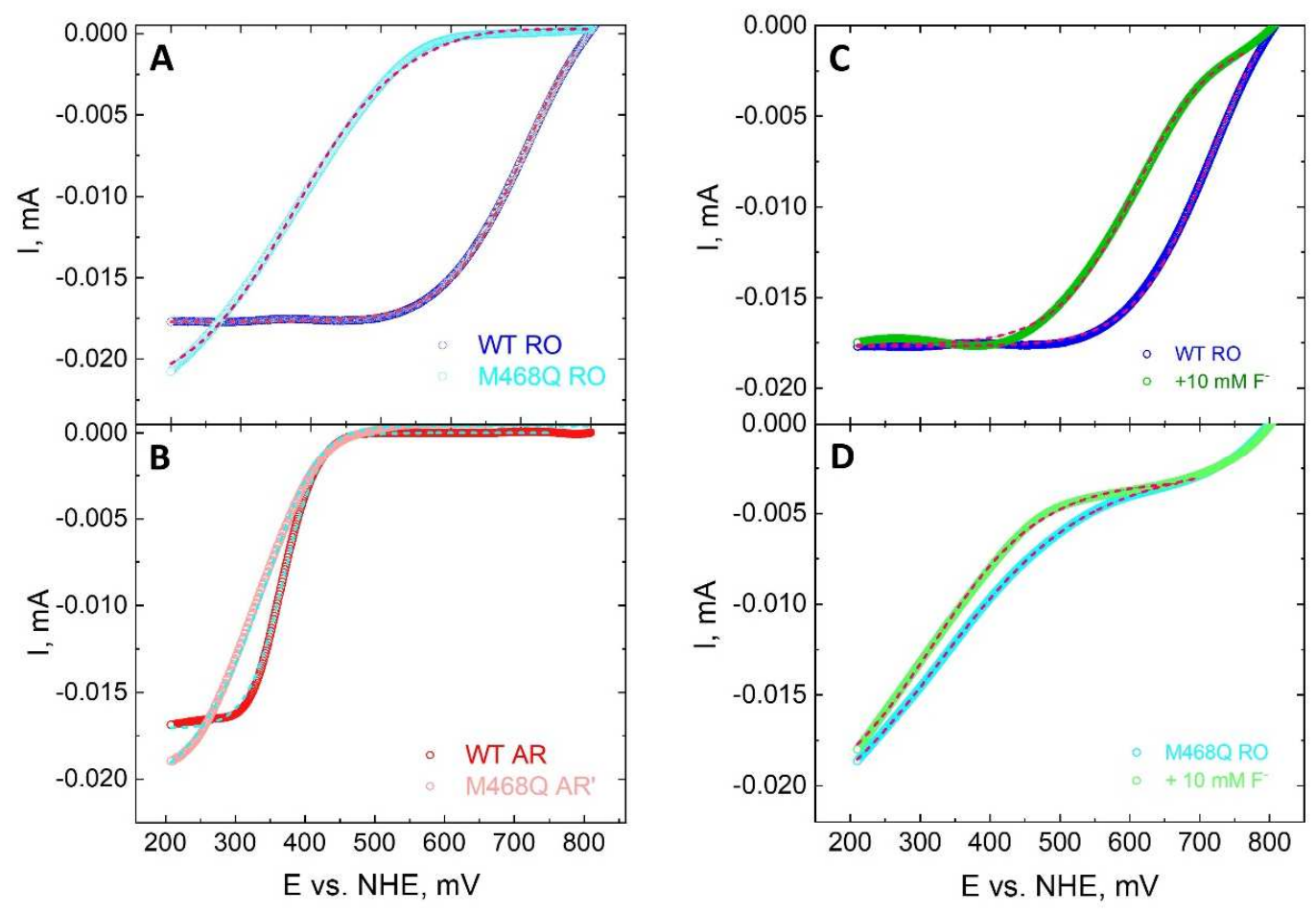

Figure 6. Fits of the experimental linear sweep voltammograms of CNFs-modified SPGEs with immobilized MoBOD variants to Scheme 1 (magenta dashed lines) for RO and RO treated with $\mathrm{F}^{-}$ and Scheme 2 (cyan dashed lines) for AR: (A) Wild-type (blue) vs. M468Q (light blue) in their resting oxidized forms. (B) Wild type (red) vs. M468Q (pink) in their alternative resting forms. (C) Wild-type in the resting oxidized form before (blue) and after (green) addition of $10 \mathrm{mM} \mathrm{NaF}$. (D) M468Q (light blue) in the resting oxidized form before (light blue) and after (light green) addition of $10 \mathrm{mM} \mathrm{NaF} . \mathrm{O}_{2}$-saturated Mcllvaine buffer ( $\mathrm{pH} 5.0$ ) at $23{ }^{\circ} \mathrm{C}$, scan rate $2 \mathrm{mV} \mathrm{s}^{-1}$, negative current represents reduction. 


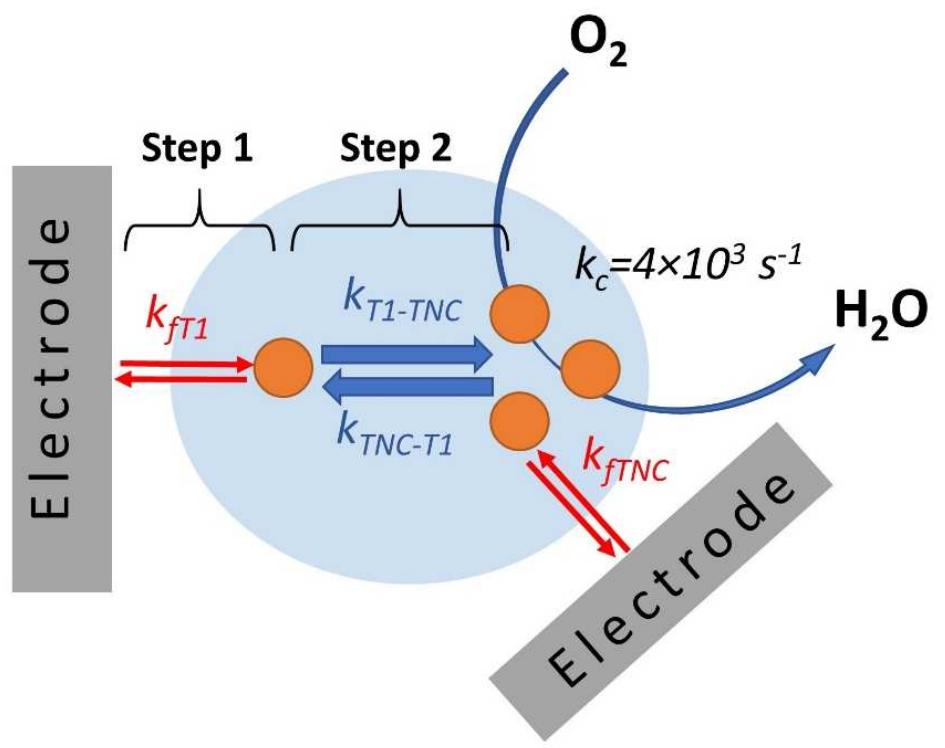

Figure 7. Schematic representation of possible orientations of a protein molecule for the electron transfer to the TNC. Orange circles depict the T1 $\mathrm{Cu}$ site and the TNC. 


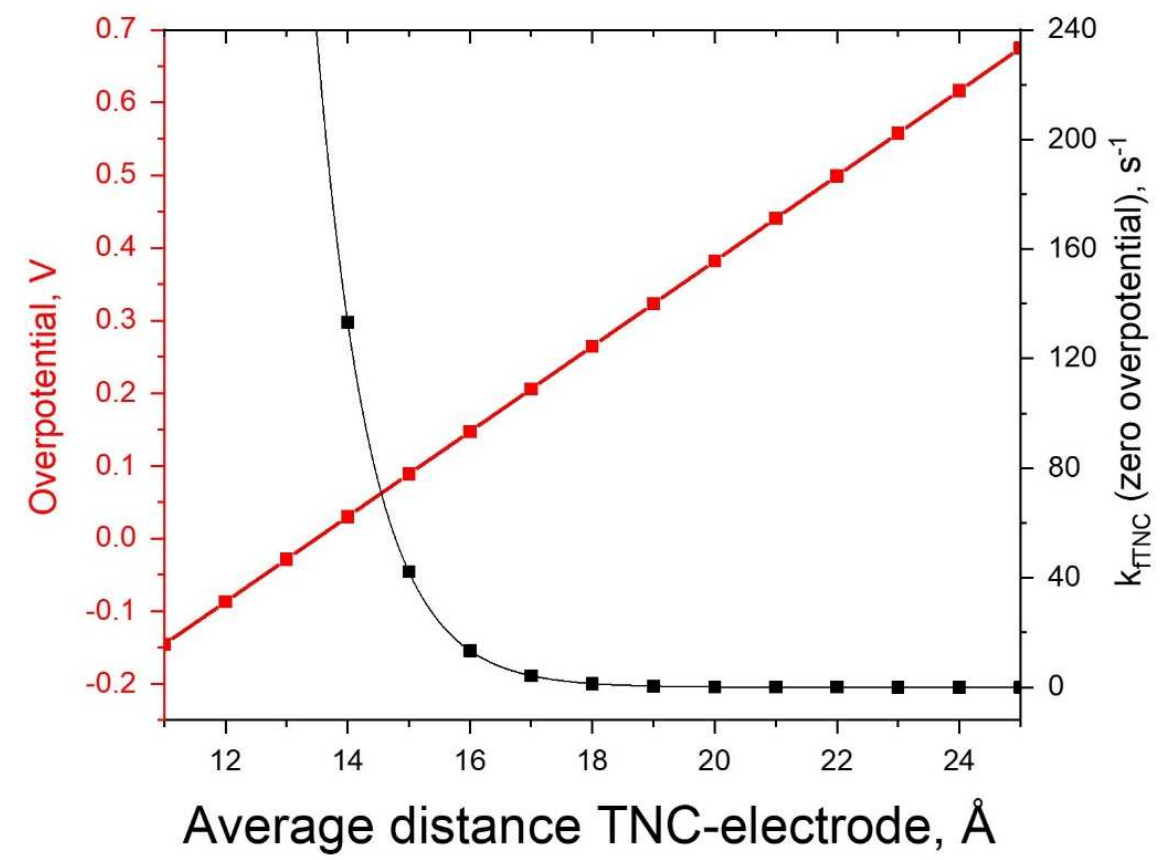

Figure 8. Dependence of the overpotential required for DET to the NI TNC to exceed T1 to NI TNC intramolecular electron transfer on the average distance between the electrode and the TNC (red) and dependence of the rate constant for direct electron transfer to the NI TNC $\left(k_{f T N C}\right)$ at zero overpotential on the average distance between the TNC and the electrode (black). 


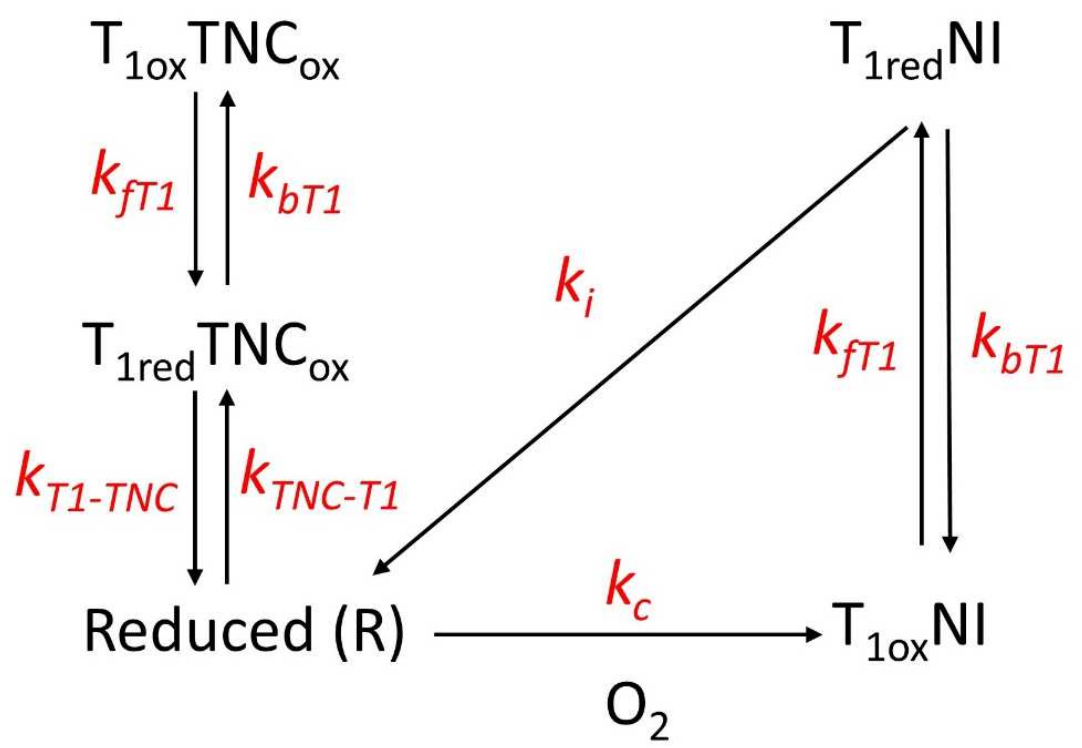

Scheme 1. Kinetic scheme of dioxygen reduction to water by a high potential MCO with the interfacial ET through the T1 site. 


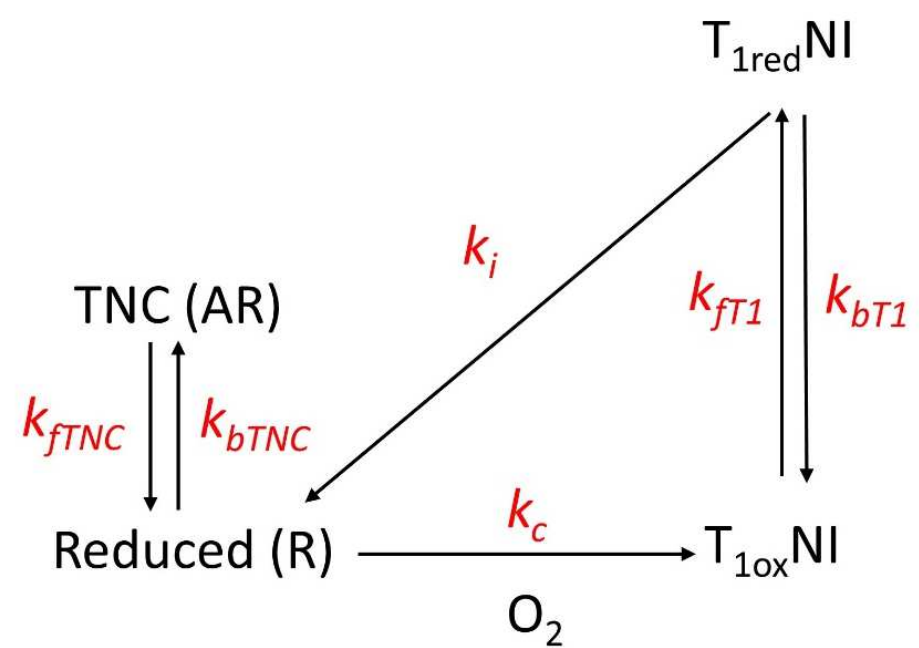

Scheme 2. Kinetic scheme of dioxygen reduction to water by a high potential MCO with the first interfacial ET to the AR form of the TNC and following ETs through the T1 site. 


\section{REFERENCES}

(1) Solomon, E. I.; Heppner, D. E.; Johnston, E. M.; Ginsbach, J. W.; Cirera, J.; Qayyum, M.; KieberEmmons, M. T.; Kjaergaard, C. H.; Hadt, R. G.; Tian, L. Copper Active Sites in Biology. Chem. Rev. 2014, 114 (7), 3659-3853. https://doi.org/10.1021/cr400327t.

(2) Jones, S. M.; Solomon, E. I. Electron Transfer and Reaction Mechanism of Laccases. Cell. Mol. Life Sci. 2015, 72 (5), 869-883. https://doi.org/10.1007/s00018-014-1826-6.

(3) Solomon, E. I.; Sundaram, U. M.; Machonkin, T. E. Multicopper Oxidases and Oxygenases. Chem. Rev. 1996, 96 (7), 2563-2606. https://doi.org/10.1021/cr950046o.

(4) Morozova, O. V.; Shumakovich, G. P.; Gorbacheva, M. A.; Shleev, S. V.; Yaropolov, A. I. "Blue" Laccases. Biochem. Mosc. 2007, 72 (10), 1136-1150. https://doi.org/10.1134/S0006297907100112.

(5) Shleev, S.; Tkac, J.; Christenson, A.; Ruzgas, T.; Yaropolov, A. I.; Whittaker, J. W.; Gorton, L. Direct Electron Transfer between Copper-Containing Proteins and Electrodes. Biosens. Bioelectron. 2005, 20 (12), 2517-2554. https://doi.org/10.1016/j.bios.2004.10.003.

(6) Mano, N.; de Poulpiquet, A. O2 Reduction in Enzymatic Biofuel Cells. Chem. Rev. 2018, 118 (5), 2392-2468. https://doi.org/10.1021/acs.chemrev.7b00220.

(7) Schweiger, H.; Vayner, E.; Anderson, A. B. Why Is There Such a Small Overpotential for $\mathrm{O2}$ Electroreduction by Copper Laccase? Electrochem. Solid-State Lett. 2005, 8 (11), A585. https://doi.org/10.1149/1.2050547.

(8) Shin, W.; Sundaram, U. M.; Cole, J. L.; Zhang, H. H.; Hedman, B.; Hodgson, K. O.; Solomon, E. I. Chemical and Spectroscopic Definition of the Peroxide-Level Intermediate in the Multicopper Oxidases: Relevance to the Catalytic Mechanism of Dioxygen Reduction to Water. J. Am. Chem. Soc. 1996, 118 (13), 3202-3215. https://doi.org/10.1021/ja953621e.

(9) Lee, S.-K.; George, S. D.; Antholine, W. E.; Hedman, B.; Hodgson, K. O.; Solomon, E. I. Nature of the Intermediate Formed in the Reduction of $\mathrm{O} 2$ to $\mathrm{H} 2 \mathrm{O}$ at the Trinuclear Copper Cluster Active Site in Native Laccase. J. Am. Chem. Soc. 2002, 124 (21), 6180-6193. https://doi.org/10.1021/ja0114052.

(10) Andréasson, L. E.; Reinhammar, B. The Mechanism of Electron Transfer in Laccase-Catalysed Reactions. Biochim. Biophys. Acta 1979, 568 (1), 145-156. https://doi.org/10.1016/00052744(79)90282-1.

(11) Sekretaryova, A.; Jones, S. M.; Solomon, E. I. O2 Reduction to Water by High Potential Multicopper Oxidases: Contributions of the T1 Copper Site Potential and the Local Environment of the Trinuclear Copper Cluster. J. Am. Chem. Soc. 2019, 141 (28), 11304-11314. https://doi.org/10.1021/jacs.9b05230.

(12) Yoon, J.; Solomon, E. I. Electronic Structure of the Peroxy Intermediate and Its Correlation to the Native Intermediate in the Multicopper Oxidases: Insights into the Reductive Cleavage of the O-O Bond. J. Am. Chem. Soc. 2007, 129 (43), 13127-13136. https://doi.org/10.1021/ja073947a.

(13) Hadt, R. G.; Gorelsky, S. I.; Solomon, E. I. Anisotropic Covalency Contributions to Superexchange Pathways in Type One Copper Active Sites. J. Am. Chem. Soc. 2014, 136 (42), 15034-15045. https://doi.org/10.1021/ja508361h.

(14) Palmer, A. E.; Lee, S. K.; Solomon, E. I. Decay of the Peroxide Intermediate in Laccase: Reductive Cleavage of the O-O Bond. J. Am. Chem. Soc. 2001, 123 (27), 6591-6599. https://doi.org/10.1021/ja010365z.

(15) Heppner, D. E.; Kjaergaard, C. H.; Solomon, E. I. Mechanism of the Reduction of the Native Intermediate in the Multicopper Oxidases: Insights into Rapid Intramolecular Electron Transfer in Turnover. J. Am. Chem. Soc. 2014, 136 (51), 17788-17801. https://doi.org/10.1021/ja509150j. 
(16) Tian, S.; Jones, S. M.; Jose, A.; Solomon, E. I. Chloride Control of the Mechanism of Human Serum Ceruloplasmin (Cp) Catalysis. J. Am. Chem. Soc. 2019, 141 (27), 10736-10743. https://doi.org/10.1021/jacs.9b03661.

(17) Jones, S. M.; Heppner, D. E.; Vu, K.; Kosman, D. J.; Solomon, E. I. Rapid Decay of the Native Intermediate in the Metallooxidase Fet3p Enables Controlled Fell Oxidation for Efficient Metabolism. J. Am. Chem. Soc. 2020, 142 (22), 10087-10101. https://doi.org/10.1021/jacs.0c02384.

(18) Quintanar, L.; Yoon, J.; Aznar, C. P.; Palmer, A. E.; Andersson, K. K.; Britt, R. D.; Solomon, E. I. Spectroscopic and Electronic Structure Studies of the Trinuclear Cu Cluster Active Site of the Multicopper Oxidase Laccase: Nature of Its Coordination Unsaturation. J. Am. Chem. Soc. 2005, 127 (40), 13832-13845. https://doi.org/10.1021/ja0421405.

(19) Reinhammar, B. R. M. Oxidation-Reduction Potentials of the Electron Acceptors in Laccases and Stellacyanin. Biochim. Biophys. Acta BBA - Bioenerg. 1972, 275 (2), 245-259. https://doi.org/10.1016/0005-2728(72)90045-X.

(20) Kepp, K. P. Halide Binding and Inhibition of Laccase Copper Clusters: The Role of Reorganization Energy. Inorg. Chem. 2015, 54 (2), 476-483. https://doi.org/10.1021/ic5021466.

(21) Antiochia, R.; Oyarzun, D.; Sánchez, J.; Tasca, F. Comparison of Direct and Mediated Electron Transfer for Bilirubin Oxidase from Myrothecium Verrucaria. Effects of Inhibitors and Temperature on the Oxygen Reduction Reaction. Catalysts 2019, 9 (12), 1056. https://doi.org/10.3390/catal9121056.

(22) Kjaergaard, C. H.; Durand, F.; Tasca, F.; Qayyum, M. F.; Kauffmann, B.; Gounel, S.; Suraniti, E.; Hodgson, K. O.; Hedman, B.; Mano, N.; Solomon, E. I. Spectroscopic and Crystallographic Characterization of "Alternative Resting" and "Resting Oxidized" Enzyme Forms of Bilirubin Oxidase: Implications for Activity and Electrochemical Behavior of Multicopper Oxidases. J. Am. Chem. Soc. 2012, 134 (12), 5548-5551. https://doi.org/10.1021/ja211872j.

(23) V. Hexter, S.; F. Esterle, T.; A. Armstrong, F. A Unified Model for Surface Electrocatalysis Based on Observations with Enzymes. Phys. Chem. Chem. Phys. 2014, 16 (24), 11822-11833. https://doi.org/10.1039/C3CP55230F.

(24) Christenson, A.; Shleev, S.; Mano, N.; Heller, A.; Gorton, L. Redox Potentials of the Blue Copper Sites of Bilirubin Oxidases. Biochim. Biophys. Acta BBA - Bioenerg. 2006, 1757 (12), 1634-1641. https://doi.org/10.1016/j.bbabio.2006.08.008.

(25) Kamitaka, Y.; Tsujimura, S.; Kataoka, K.; Sakurai, T.; Ikeda, T.; Kano, K. Effects of Axial Ligand Mutation of the Type I Copper Site in Bilirubin Oxidase on Direct Electron Transfer-Type Bioelectrocatalytic Reduction of Dioxygen. J. Electroanal. Chem. 2007, 601 (1-2), 119-124. https://doi.org/10.1016/j.jelechem.2006.10.035.

(26) Yaropolov, A. I.; Kharybin, A. N.; Emnéus, J.; Marko-Varga, G.; Gorton, L. Electrochemical Properties of Some Copper-Containing Oxidases. Bioelectrochem. Bioenerg. 1996, 40 (1), 49-57. https://doi.org/10.1016/0302-4598(96)01919-8.

(27) Gupta, G.; Rajendran, V.; Atanassov, P. Bioelectrocatalysis of Oxygen Reduction Reaction by Laccase on Gold Electrodes. Electroanalysis 2004, 16 (13-14), 1182-1185. https://doi.org/10.1002/elan.200403010.

(28) Dronov, R.; Kurth, D. G.; Scheller, F. W.; Lisdat, F. Direct and Cytochrome c Mediated Electrochemistry of Bilirubin Oxidase on Gold. Electroanalysis 2007, 19 (15), 1642-1646. https://doi.org/10.1002/elan.200703900.

(29) Pita, M.; Shleev, S.; Ruzgas, T.; Fernández, V. M.; Yaropolov, A. I.; Gorton, L. Direct Heterogeneous Electron Transfer Reactions of Fungal Laccases at Bare and Thiol-Modified Gold Electrodes. Electrochem. Commun. 2006, 8 (5), 747-753.

https://doi.org/10.1016/j.elecom.2006.03.008. 
(30) de Poulpiquet, A.; Kjaergaard, C. H.; Rouhana, J.; Mazurenko, I.; Infossi, P.; Gounel, S.; Gadiou, R.; Giudici-Orticoni, M. T.; Solomon, E. I.; Mano, N.; Lojou, E. Mechanism of Chloride Inhibition of Bilirubin Oxidases and Its Dependence on Potential and PH. ACS Catal. 2017, 7 (6), 3916-3923. https://doi.org/10.1021/acscatal.7b01286.

(31) Dagys, M.; Laurynènas, A.; Ratautas, D.; Kulys, J.; Vidžiūnaitè, R.; Talaikis, M.; Niaura, G.; Marcinkevičienè, L.; Meškys, R.; Shleev, S. Oxygen Electroreduction Catalysed by Laccase Wired to Gold Nanoparticles via the Trinuclear Copper Cluster. Energy Environ. Sci. 2017, 10 (2), 498502. https://doi.org/10.1039/C6EE02232D.

(32) Vaz-Dominguez, C.; Campuzano, S.; Rüdiger, O.; Pita, M.; Gorbacheva, M.; Shleev, S.; Fernandez, V. M.; De Lacey, A. L. Laccase Electrode for Direct Electrocatalytic Reduction of $\mathrm{O} 2$ to $\mathrm{H} 2 \mathrm{O}$ with High-Operational Stability and Resistance to Chloride Inhibition. Biosens. Bioelectron. 2008, 24 (4), 531-537. https://doi.org/10.1016/j.bios.2008.05.002.

(33) Durand, F.; Gounel, S.; Kjaergaard, C. H.; Solomon, E. I.; Mano, N. Bilirubin Oxidase from Magnaporthe Oryzae: An Attractive New Enzyme for Biotechnological Applications. Appl. Microbiol. Biotechnol. 2012, 96 (6), 1489-1498. https://doi.org/10.1007/s00253-012-3926-2.

(34) Durand, F.; Kjaergaard, C. H.; Suraniti, E.; Gounel, S.; Hadt, R. G.; Solomon, E. I.; Mano, N. Bilirubin Oxidase from Bacillus Pumilus: A Promising Enzyme for the Elaboration of Efficient Cathodes in Biofuel Cells. Biosens. Bioelectron. 2012, 35 (1), 140-146.

https://doi.org/10.1016/j.bios.2012.02.033.

(35) Gounel, S.; Rouhana, J.; Stines-Chaumeil, C.; Cadet, M.; Mano, N. Increasing the Catalytic Activity of Bilirubin Oxidase from Bacillus Pumilus: Importance of Host Strain and Chaperones Proteins. J. Biotechnol. 2016, 230, 19-25. https://doi.org/10.1016/j.jbiotec.2016.04.035.

(36) Felsenfeld, G. The Determination of Cuprous Ion in Copper Proteins. Arch. Biochem. Biophys. 1960, 87 (2), 247-251. https://doi.org/10.1016/0003-9861(60)90168-5.

(37) Carithers, R. P.; Palmer, G. Characterization of the Potentiometric Behavior of Soluble Cytochrome Oxidase by Magnetic Circular Dichroism. Evidence in Support of Heme-Heme Interaction. J. Biol. Chem. 1981, 256 (15), 7967-7976. https://doi.org/10.1016/S00219258(18)43374-1.

(38) Stoll, S.; Schweiger, A. EasySpin, a Comprehensive Software Package for Spectral Simulation and Analysis in EPR. J. Magn. Reson. 2006, 178 (1), 42-55. https://doi.org/10.1016/j.jmr.2005.08.013.

(39) Machonkin, T. E.; Solomon, E. I. The Thermodynamics, Kinetics, and Molecular Mechanism of Intramolecular Electron Transfer in Human Ceruloplasmin. J. Am. Chem. Soc. 2000, 122 (50), 12547-12560. https://doi.org/10.1021/ja002339r.

(40) Palmer, A. E.; Quintanar, L.; Severance, S.; Wang, T.-P.; Kosman, D. J.; Solomon, E. I. Spectroscopic Characterization and $\mathrm{O} 2$ Reactivity of the Trinuclear Cu Cluster of Mutants of the Multicopper Oxidase Fet3p. Biochemistry 2002, 41 (20), 6438-6448. https://doi.org/10.1021/bi011979j.

(41) Blackburn, N. J.; Ralle, M.; Hassett, R.; Kosman, D. J. Spectroscopic Analysis of the Trinuclear Cluster in the Fet3 Protein from Yeast, a Multinuclear Copper Oxidase. Biochemistry 2000, 39 (9), 2316-2324. https://doi.org/10.1021/bi992334a.

(42) Cole, J. L.; Clark, P. A.; Solomon, E. I. Spectroscopic and Chemical Studies of the Laccase Trinuclear Copper Active Site: Geometric and Electronic Structure. J. Am. Chem. Soc. 1990, 112 (26), 95349548. https://doi.org/10.1021/ja00182a013.

(43) Kjaergaard, C. H.; Jones, S. M.; Gounel, S.; Mano, N.; Solomon, E. I. Two-Electron Reduction versus One-Electron Oxidation of the Type 3 Pair in the Multicopper Oxidases. J. Am. Chem. Soc. 2015, 137 (27), 8783-8794. https://doi.org/10.1021/jacs.5b04136.

(44) DeBeer George, S.; Basumallick, L.; Szilagyi, R. K.; Randall, D. W.; Hill, M. G.; Nersissian, A. M.; Valentine, J. S.; Hedman, B.; Hodgson, K. O.; Solomon, E. I. Spectroscopic Investigation of 
Stellacyanin Mutants: Axial Ligand Interactions at the Blue Copper Site. J. Am. Chem. Soc. 2003, 125 (37), 11314-11328. https://doi.org/10.1021/ja035802j.

(45) Shimizu, A.; Sasaki, T.; Kwon, J. H.; Odaka, A.; Satoh, T.; Sakurai, N.; Sakurai, T.; Yamaguchi, S.; Samejima, T. Site-Directed Mutagenesis of a Possible Type 1 Copper Ligand of Bilirubin Oxidase; a Met467GIn Mutant Shows Stellacyanin-Like Properties. J. Biochem. (Tokyo) 1999, 125 (4), 662668.

(46) Hall, J. F.; Kanbi, L. D.; Strange, R. W.; Hasnain, S. S. Role of the Axial Ligand in Type 1 Cu Centers Studied by Point Mutations of Met148 in Rusticyanin. Biochemistry 1999, 38 (39), 12675-12680. https://doi.org/10.1021/bi990983g.

(47) Hibino, T.; Lee, B. H.; Takabe, T.; Takabe, T. Expression and Characterization of Met92Gln Mutant Plastocyanin from Silene Pratensis. J. Biochem. (Tokyo) 1995, 117 (1), 101-106.

(48) Romero, A.; Hoitink, C. W. G.; Nar, H.; Huber, R.; Messerschmidt, A.; Canters, G. W. X-Ray Analysis and Spectroscopic Characterization of M121Q Azurin: A Copper Site Model for Stellacyanin. J. Mol. Biol. 1993, 229 (4), 1007-1021. https://doi.org/10.1006/jmbi.1993.1101.

(49) Scott, S. L.; Chen, W. J.; Bakac, A.; Espenson, J. H. Spectroscopic Parameters, Electrode Potentials, Acid Ionization Constants, and Electron Exchange Rates of the 2,2'-Azinobis(3-

Ethylbenzothiazoline-6-Sulfonate) Radicals and lons. J. Phys. Chem. 1993, 97 (25), 6710-6714. https://doi.org/10.1021/j100127a022.

(50) Quintanar, L.; Stoj, C.; Wang, T.-P.; Kosman, D. J.; Solomon, E. I. Role of Aspartate 94 in the Decay of the Peroxide Intermediate in the Multicopper Oxidase Fet3p. Biochemistry 2005, 44 (16), 6081-6091. https://doi.org/10.1021/bi047379c.

(51) Gentil, S.; Carrière, M.; Cosnier, S.; Gounel, S.; Mano, N.; Le Goff, A. Direct Electrochemistry of Bilirubin Oxidase from Magnaporthe Orizae on Covalently-Functionalized MWCNT for the Design of High-Performance Oxygen-Reducing Biocathodes. Chem. - Eur. J. 2018, 24 (33), 8404-8408. https://doi.org/10.1002/chem.201800774.

(52) Agbo, P.; Heath, J. R.; Gray, H. B. Modeling Dioxygen Reduction at Multicopper Oxidase Cathodes. J. Am. Chem. Soc. 2014, 136 (39), 13882-13887. https://doi.org/10.1021/ja5077519.

(53) Langen, R.; Jensen, G. M.; Jacob, U.; Stephens, P. J.; Warshel, A. Protein Control of Iron-Sulfur Cluster Redox Potentials. J. Biol. Chem. 1992, 267 (36), 25625-25627.

(54) Fourmond, V.; Wiedner, E. S.; Shaw, W. J.; Léger, C. Understanding and Design of Bidirectional and Reversible Catalysts of Multielectron, Multistep Reactions. J. Am. Chem. Soc. 2019, 141 (28), 11269-11285. https://doi.org/10.1021/jacs.9b04854.

(55) Palmer, A. E.; Randall, D. W.; Xu, F.; Solomon, E. I. Spectroscopic Studies and Electronic Structure Description of the High Potential Type 1 Copper Site in Fungal Laccase: Insight into the Effect of the Axial Ligand. J. Am. Chem. Soc. 1999, 121 (30), 7138-7149. https://doi.org/10.1021/ja991087v.

(56) Bard, A. J.; Faulkner, L. R. Electrochemical Methods: Fundamentals and Applications, 2nd edition.; Willey, 2000, 92-107.

(57) Léger, C.; Jones, A. K.; Albracht, S. P. J.; Armstrong, F. A. Effect of a Dispersion of Interfacial Electron Transfer Rates on Steady State Catalytic Electron Transport in [NiFe]-Hydrogenase and Other Enzymes. J. Phys. Chem. B 2002, 106 (50), 13058-13063.

https://doi.org/10.1021/jp0265687.

(58) Augustine, A. J.; Kragh, M. E.; Sarangi, R.; Fujii, S.; Liboiron, B. D.; Stoj, C. S.; Kosman, D. J.; Hodgson, K. O.; Hedman, B.; Solomon, E. I. Spectroscopic Studies of Perturbed T1 Cu Sites in the Multicopper Oxidases Saccharomyces Cerevisiae Fet3p and Rhus Vernicifera Laccase: Allosteric Coupling between the T1 and Trinuclear Cu Sites. Biochemistry 2008, 47 (7), 2036-2045. https://doi.org/10.1021/bi7020052. 
(59) Shleev, S.; Andoralov, V.; Falk, M.; Reimann, C. T.; Ruzgas, T.; Srnec, M.; Ryde, U.; Rulíšek, L. On the Possibility of Uphill Intramolecular Electron Transfer in Multicopper Oxidases:

Electrochemical and Quantum Chemical Study of Bilirubin Oxidase. Electroanalysis 2012, 24 (7), 1524-1540. https://doi.org/10.1002/elan.201200188.

(60) Marcus, R. A. Electron Transfer Reactions in Chemistry. Theory and Experiment. Rev. Mod. Phys. 1993, 65 (3), 599-610. https://doi.org/10.1103/RevModPhys.65.599.

(61) Li, Y.; Zhang, J.; Huang, X.; Wang, T. Construction and Direct Electrochemistry of Orientation Controlled Laccase Electrode. Biochem. Biophys. Res. Commun. 2014, 446 (1), 201-205. https://doi.org/10.1016/j.bbrc.2014.02.084.

(62) Ramírez, P.; Mano, N.; Andreu, R.; Ruzgas, T.; Heller, A.; Gorton, L.; Shleev, S. Direct Electron Transfer from Graphite and Functionalized Gold Electrodes to T1 and T2/T3 Copper Centers of Bilirubin Oxidase. Biochim. Biophys. Acta BBA - Bioenerg. 2008, 1777 (10), 1364-1369. https://doi.org/10.1016/j.bbabio.2008.06.010.

(63) Al-Lolage, F. A.; Bartlett, P. N.; Gounel, S.; Staigre, P.; Mano, N. Site-Directed Immobilization of Bilirubin Oxidase for Electrocatalytic Oxygen Reduction. ACS Catal. 2019, 9 (3), 2068-2078. https://doi.org/10.1021/acscatal.8b04340.

(64) Sekretaryova, A. N.; Vagin, M. Yu.; Turner, A. P. F.; Eriksson, M. Electrocatalytic Currents from Single Enzyme Molecules. J. Am. Chem. Soc. 2016, 138 (8), 2504-2507. https://doi.org/10.1021/jacs.5b13149. 
TOC graphic

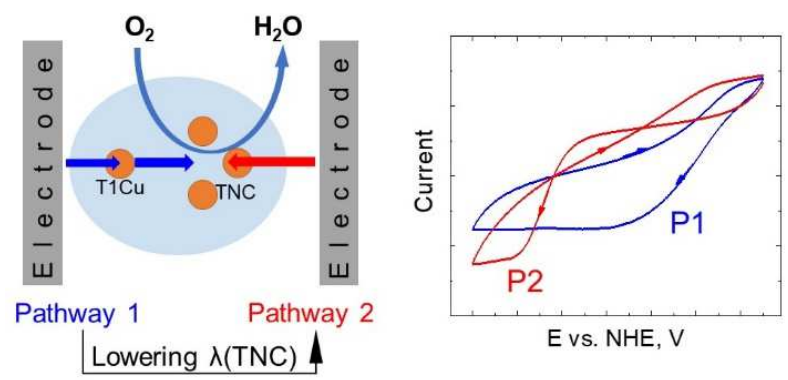




\title{
Supporting Information
}

\section{Electron Transfer to the Trinuclear Copper Cluster in Electrocatalysis by the Multicopper Oxidases}

\author{
Alina Sekretareva, ${ }^{\dagger} \#, *$ Shiliang Tian,${ }^{\dagger}$ Sébastien Gounel, $"$ Nicolas Mano,,$\AA$ \\ Edward I. Solomon ${ }^{\dagger, * * *}$ \\ ${ }^{\dagger}$ Department of Chemistry, Stanford University, Stanford, California 94305, United States \\ $¥$ SLAC National Accelerator Laboratory, Stanford University, California 94025, United States \\ \# Department of Chemistry, Ångström Laboratory, Uppsala University, SE-75120, Uppsala, \\ Sweden \\ $\S$ CNRS, CRPP, UPR 8641, 33600 Pessac, France \\ "Université de Bordeaux, CRPP, UMR5031, 33600 Pessac, France
}




\section{Supporting Note 1. Derivation of the cathodic current expression for kinetic Scheme 1}

The overall rate of the process can be described as follows:

$r=k_{c}[R]$

For simplicity of expressions, the concentrations of different forms are denoted as:

$$
\begin{aligned}
& \mathrm{A}-\left[\mathrm{T}_{1 \mathrm{ox}} \mathrm{TNC}_{\mathrm{ox}}\right] \\
& \mathrm{B}-\left[\mathrm{T}_{1 \text { red }} \mathrm{TNC}_{\mathrm{ox}}\right] \\
& \mathrm{C}-[\mathrm{R}], \text { fully reduced form } \\
& \mathrm{D}-\left[\mathrm{T}_{1 \mathrm{ox}} \mathrm{NI}\right] \\
& \mathrm{E}-\left[\mathrm{T}_{1 \mathrm{red}} \mathrm{NI}\right]
\end{aligned}
$$

Surface concentrations of all forms can be expressed through the following system of equations:

$\frac{d A}{d t}=k_{b T 1} B-k_{f T 1} A=0$

$\frac{d B}{d t}=k_{f T 1} A+k_{T N C-T 1} C-k_{b T 1} B-k_{T 1-T N C} B=0$

$\frac{d C}{d t}=k_{T 1-T N C} B+k_{i} E-k_{T N C-T 1} C-k_{C} C=0$

$\frac{d D}{d t}=k_{c} C+k_{b T 1} E-k_{f T 1} D=0$

$\frac{d E}{d t}=k_{f T 1} D-k_{b T 1} E-k_{i} E=0$

Under the steady-state conditions, the surface concentrations of various forms can be written as:

$$
\begin{aligned}
& B=\frac{k_{f T 1}}{k_{b} T 1} A \\
& C=\frac{k_{T 1-T N C} k_{f T 1}}{k_{T N C-T 1} k_{b T 1}} A \\
& E=\frac{k_{T 1-T N C} k_{c} k_{f T 1}}{k_{T N C-T 1} k_{b T 1} k_{i}} A \\
& D=\frac{k_{T 1-T N C} k_{C}\left(k_{b T 1}+k_{i}\right)}{k_{T N C-T 1} k_{b T 1} k_{i}} A
\end{aligned}
$$


The total enzyme concentration, $\Gamma$, can be written as a sum of the concentrations of all the intermediate forms present during catalytic cycling:

$\Gamma=A+B+C+D+E=$

$=A\left(1+\frac{k_{f T 1}}{k_{b T 1}}+\frac{k_{T 1-T N C} k_{f T 1}}{k_{T N C-T 1} k_{b T 1}}+\frac{k_{T 1-T N C} k_{C}\left(k_{b T 1}+k_{i}\right)}{k_{T N C-T 1} k_{b T 1} k_{i}}+\frac{k_{T 1-T N C} k_{c} k_{f T 1}}{k_{T N C-T 1} k_{b T 1} k_{i}}\right)$

$A=\frac{\Gamma\left(k_{T N C-T 1} k_{b T 1} k_{i}\right)}{k_{T 1-T N C} k_{C}\left(k_{b T 1}+k_{i}+k_{f T 1}\right)+k_{i}\left(k_{T N C-T 1} k_{b T 1}+k_{T N C-T 1} k_{f T 1}+k_{T 1-T N C} k_{f T 1}\right)}$

The rate of the equation then can be expressed as follows:

$$
\begin{gathered}
r=k_{C} \times \frac{k_{T 1-T N C} k_{f T 1}}{k_{T N C-T 1} k_{b T 1}} \times \frac{\Gamma\left(k_{T N C-T 1} k_{b T 1} k_{i}\right)}{k_{T 1-T N C} k_{c}\left(k_{b T 1}+k_{i}+k_{f T 1}\right)+k_{i}\left(k_{T N C-T 1} k_{b T 1}+k_{T N C-T 1} k_{f T 1}+k_{T 1-T N C} k_{f T 1}\right)}= \\
=\frac{\Gamma k_{f T 1} k_{T 1-T N C} k_{c} k_{i}}{k_{i} k_{T 1-T N C}\left(k_{f T 1}+k_{i}\right)+\left(k_{C} k_{T 1-T N C}+k_{C} k_{T N C-T 1}\right)\left(k_{b T 1}+k_{f T 1}\right)}
\end{gathered}
$$

The obtained pseudo-first-order rate constant has units of $\mathrm{s}^{-1}$. 


\section{Supporting Note 2. Derivation of the cathodic current expression for kinetic Scheme 2}

For Scheme 2 the concentrations of different forms are denoted as follows:

$$
\begin{gathered}
\mathrm{F}-\left[\mathrm{TNC}^{*}\right], \text { two-electron reduced TNC form } \\
\mathrm{C}-[\mathrm{R}], \text { fully reduced form } \\
\mathrm{D}-\left[\mathrm{T}_{1 \mathrm{ox}} \mathrm{NI}\right] \\
\mathrm{E}-\left[\mathrm{T}_{1 \mathrm{red} \mathrm{NI}]}\right. \\
\frac{d F}{d t}=k_{b T N C} C-k_{f T N C} F=0 \\
\frac{d C}{d t}=k_{f T N C} F+k_{i} E-k_{b T N C} C-k_{C} C=0
\end{gathered}
$$

For concentrations of D and E, equations (s5) and (s6), respectively, are valid.

Under the steady-state conditions, the surface concentrations of various forms can be written as:

$$
\begin{aligned}
& C=\frac{k_{f T N C}}{k_{b T N C}} F \\
& E=\frac{k_{f T N C} k_{C}}{k_{b T N C} k_{i}} F \\
& D=\frac{k_{c} k_{f T N C}\left(k_{b T 1}+k_{i}\right)}{k_{b T N C} k_{f T 1} k_{i}} F
\end{aligned}
$$

The total enzyme concentration, $N$ (number of molecules), can be written as a sum of the concentrations of all the intermediate forms present during catalytic cycling:

$$
\begin{aligned}
& N=\Gamma N_{A}=F+C+D+E=F\left(1+\frac{k_{f T N C}}{k_{b T N C}}+\frac{k_{c} k_{f T N C}\left(k_{b T 1}+k_{i}\right)}{k_{b T N C} k_{f T 1} k_{i}}+\frac{k_{f T N C} k_{c}}{k_{b T N C} k_{i}}\right) \\
& F=\frac{N\left(k_{b T N C} k_{f T 1} k_{i}\right)}{\left.k_{b T N C} k_{i} k_{f T 1}+k_{i} k_{f T 1} k_{f T N C}+k_{c} k_{f T N C}\left(k_{b T 1}+k_{i}\right)+k_{f T N C} k_{c} k_{i}\right)}
\end{aligned}
$$

The rate of the equation then can be expressed as follows: 


$$
\begin{gathered}
r=k_{c} \times \frac{k_{f T N C}}{k_{b T N C}} \times \frac{N\left(k_{b T N C} k_{f T 1} k_{i}\right)}{\left.k_{b T N C} k_{i} k_{f T 1}+k_{i} k_{f T 1} k_{f T N C}+k_{c} k_{f T N C}\left(k_{b T 1}+k_{i}\right)+k_{f T N C} k_{c} k_{i}\right)}= \\
=\frac{\Gamma N_{A} k_{f T N C} k_{f T 1} k_{c} k_{i}}{k_{i} k_{b T N C} k_{f T 1}+k_{i} k_{f T N C} k_{f T 1}+k_{c} k_{f T N C} k_{f T 1}+k_{c} k_{f T N C}\left(k_{b T 1}+k_{i}\right)}
\end{gathered}
$$

The obtained pseudo-first-order rate constant has units of $\mathrm{s}^{-1}$. 


\section{Supporting Note 3. The average T1 site/electrode distance estimation}

Following the model reported by Gray and co-workers, ${ }^{52}$ the average distance can be expressed as follows:

$r=r_{0}-\frac{2}{\beta} \ln \frac{H_{D A}(r)}{H_{D A}^{0}}$

where $\beta$ is the distance-decay constant, $H_{D A}^{0}$ is the electronic coupling at the closest distance $r_{0}$. From previous studies, for the high potential MCOs immobilized on carbon electrodes, $r_{0}=3 \AA$, $H_{D A}^{0}$ is $150 \mathrm{~cm}^{-1}$, and $\beta$ is $1.1 \AA^{-1} .52$

The average distance between the primary electron acceptor and the electrode can be expressed via a probability distribution function of protein molecules orientation $(\mathrm{P}(\mathrm{r})){ }^{52}$

$<r>=\int r P(r) d r$

For the distribution where all of the possible orientations of a protein molecule on the electrode, and therefore, all distances within a certain range (between maximum, $r_{\max }$, and minimum, $r_{\min }$, values) between the electrode and the electron acceptor site occur with the same probability:

$P(r)=\frac{1}{r_{\max }-r_{\min }}$

Then the average distance is just the average of two distances:

$<r>=\frac{1}{2}\left(r_{\max }+r_{\min }\right)$ 


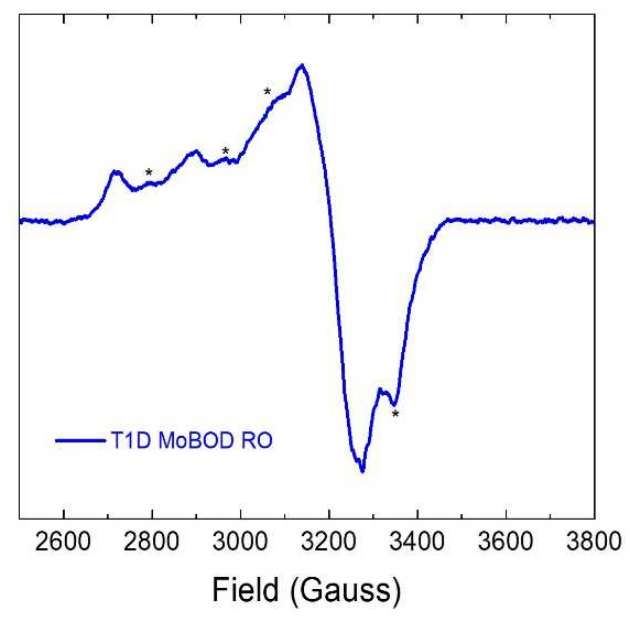

Figure S1. 77K X-band EPR spectrum of the fully oxidized T1D MoBOD. * denotes extraneously bound $\mathrm{Cu}$ in T1D MoBOD. 


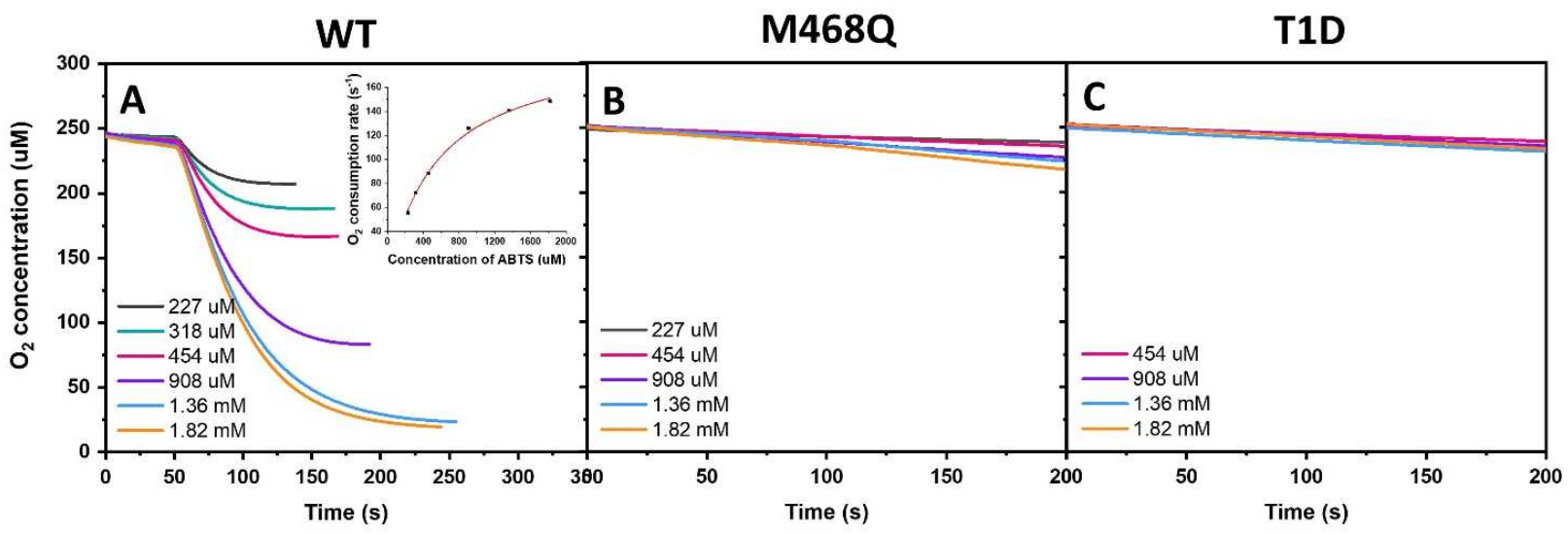

Figure S2. Dependence of enzyme activity on ABTS concentration measured by the Clark

electrode for (A) WT MoBOD, (B) M468Q MoBOD, and (C) T1D BpBOD. Measurements were performed in Mcllvaine buffer (pH 5.0) at $23{ }^{\circ} \mathrm{C}$. 


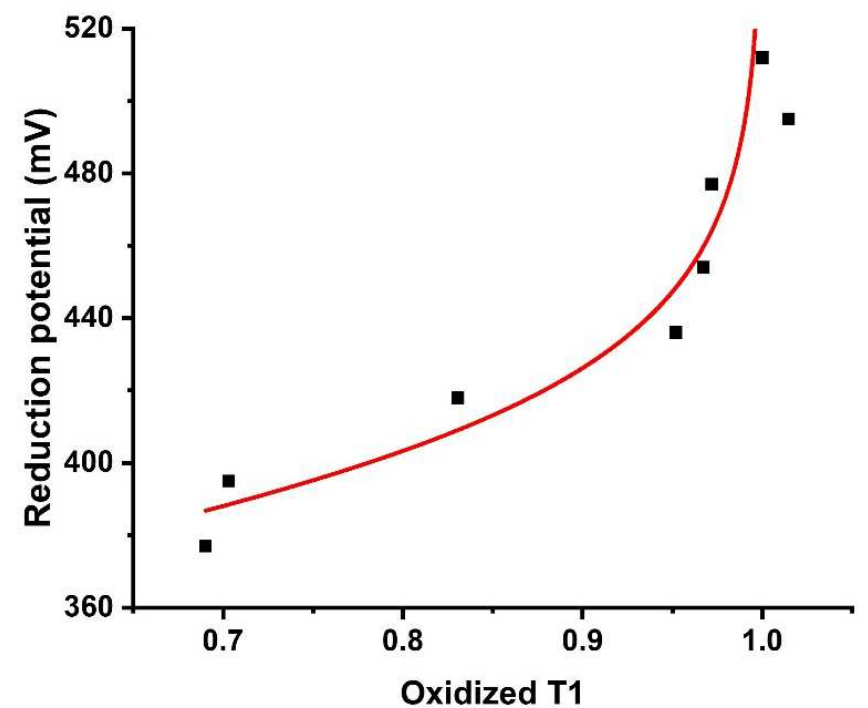

Figure S3. Potentiometric titration of the Type $1 \mathrm{Cu}$ site in M468Q MoBOD in Mcllvaine buffer (pH 5.0) at $23{ }^{\circ} \mathrm{C}$. The solid line is the fit of the data to the Nernst equation by nonlinear regression. The obtained values are $E_{T 1}{ }^{0^{\prime}}=364 \mathrm{mV}$ vs NHE, $\mathrm{n}=0.91$. 


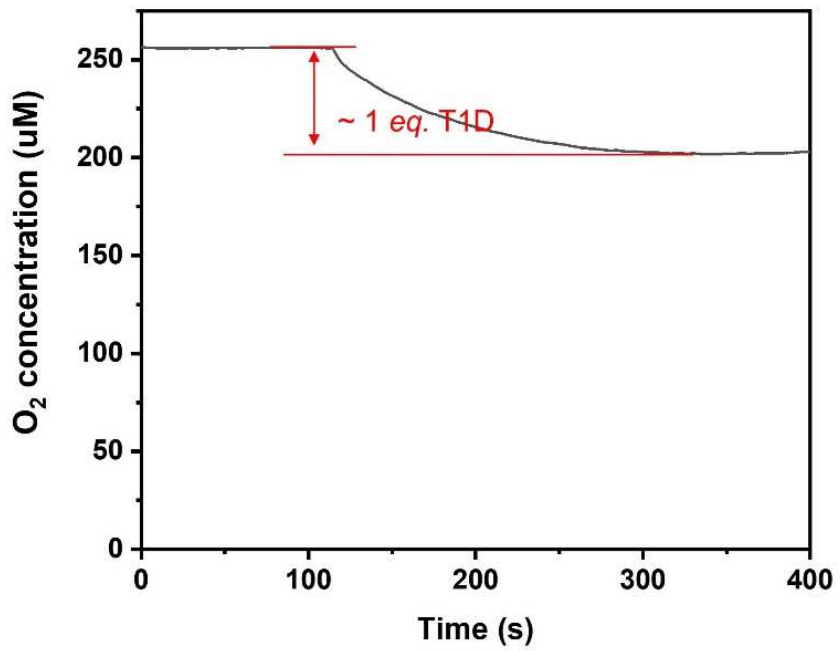

Figure S4. Catalytic activity of T1D BpBOD in fully reduced form in $1.82 \mathrm{mM}$ ABTS solution in a Mcllvaine buffer ( $\mathrm{pH} 5.0)$ at $23{ }^{\circ} \mathrm{C}$ measured by the Clark electrode. 


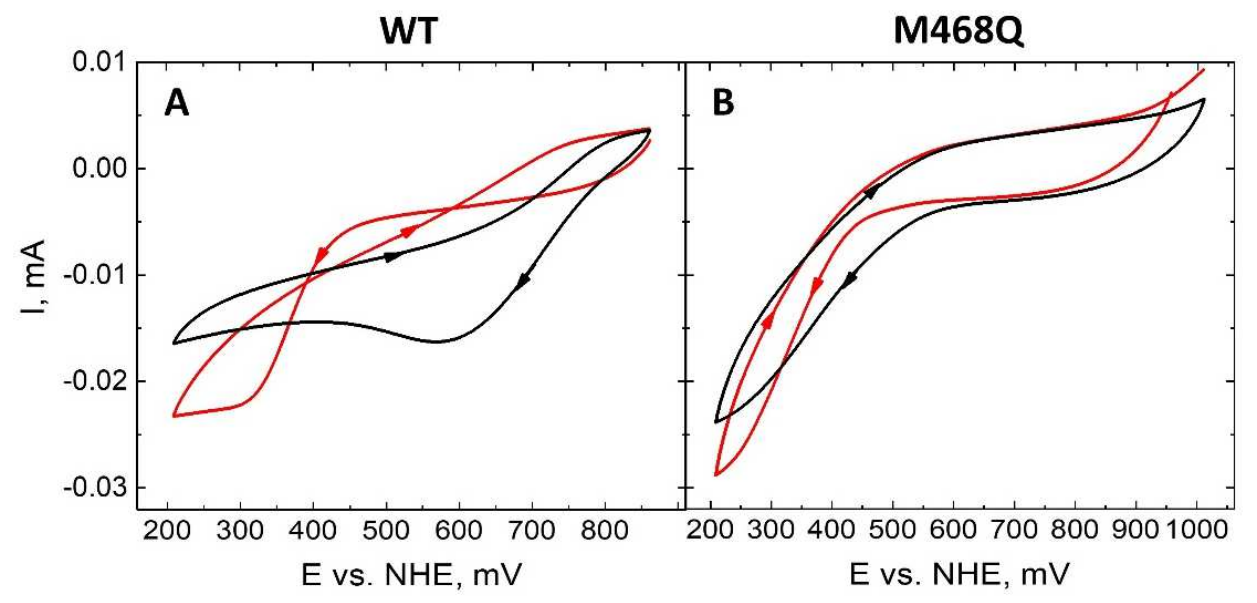

Figure S5. Cyclic voltammograms of CNFs-modified SPGEs with the immobilized MoBOD variants after addition of $100 \mathrm{mM} \mathrm{Cl}^{-}$, first cycle (red) and second cycle (black). (A) WT MoBOD, (B) M468Q MoBOD. $\mathrm{O}_{2}$-saturated Mcllvaine buffer (pH 5.0) at $23{ }^{\circ} \mathrm{C}$. Scan rate $2 \mathrm{mV} \mathrm{s}^{-1}$. Arrows indicate scan direction; negative current represents reduction. 

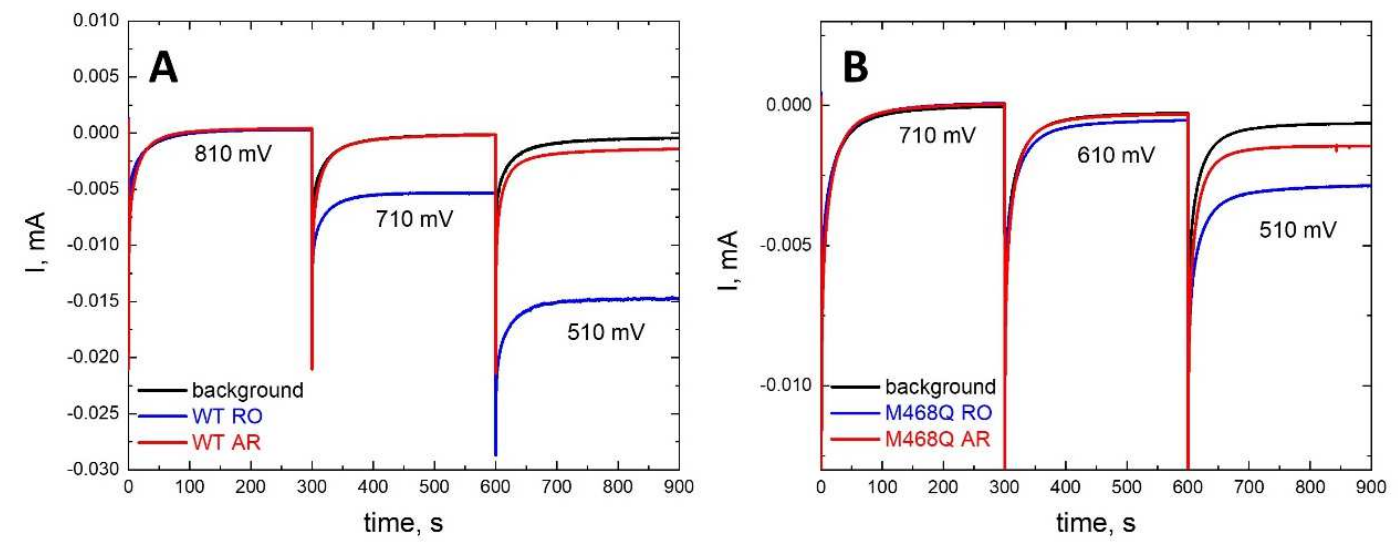

Figure S6. Potential step chrono-amperometry of CNFs-modified SPGEs with the immobilized MoBOD variants in $\mathrm{N}_{2}$-saturated buffer (black), the reoxidized enzyme before (blue) and after incubation in $100 \mathrm{mM} \mathrm{Cl}^{-}$(red) in $\mathrm{O}_{2}$-saturated Mcllvaine buffer ( $\mathrm{pH}$ 5.0) free from $\mathrm{Cl}^{-}$at $23{ }^{\circ} \mathrm{C}_{\text {. }}$ (A) WT MoBOD, (B) M468Q MoBOD. Applied potentials vs. NHE are indicated on each step. 

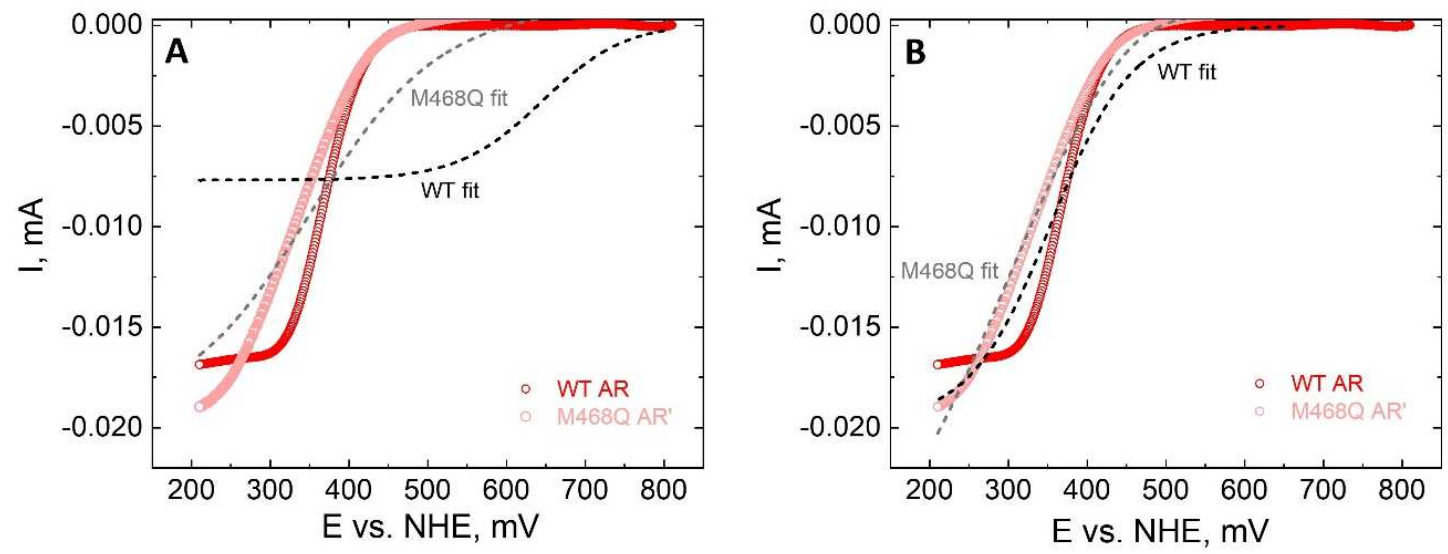

Figure S7. Fits of the experimental linear sweep voltammograms of CNFs-modified SPGEs with the immobilized wild type (red) and M468Q (pink) MoBOD variants in their alternative resting forms to Scheme 1 (black (WT) and grey (M46Q) dashed lines). (A) Best fits with the freely floating $E_{T N C}(A R)^{0^{\prime}}$ and all the remaining fitting parameters fixed to the values in Table 3. The obtained $E_{T N C}(A R)^{0^{\prime}}$ are $710 \mathrm{mV}(\mathrm{WT})$ and $800 \mathrm{mV}$ (M468Q) vs. NHE. (B) Best fits with all the fitting parameters floating within the specified range. The fitting parameters and the range tested are summarized in Table $\mathrm{S} 1 . \mathrm{O}_{2}$-saturated Mcllvaine buffer $(\mathrm{pH} 5.0)$ at $23{ }^{\circ} \mathrm{C}$, scan rate $2 \mathrm{mV} \mathrm{s}^{-1}$, negative current represents reduction. 
Table S1. Fitting parameters obtained from modeling of the LSV data for the WT and M468Q in their AR forms (Figure S7B) using Scheme 1.

\begin{tabular}{|c|c|c|}
\hline $\begin{array}{l}\text { Parameters, } \\
\text { [range tested] }\end{array}$ & WT AR & M468Q AR' \\
\hline $\mathrm{H}(\mathrm{T} 1)_{\mathrm{DA}}[0.0008-0.004], \mathrm{cm}^{-1}$ & 0.0008 & 0.0022 \\
\hline$\alpha(\mathrm{T} 1)[0-0.5]$ & 0.5 & 0.3 \\
\hline $\mathrm{k}_{\mathrm{i}}[200-400], \mathrm{s}^{-1}$ & 400 & 210 \\
\hline $\begin{array}{l}E_{T 1} 0^{\prime}[570-800 \text { for } \mathrm{WT}], \\
\quad[360-800 \text { for } \mathrm{M} 468 \mathrm{Q}], \mathrm{mV} \text { vs. } \mathrm{NHE}\end{array}$ & 570 & 360 \\
\hline$E_{T N C}{ }^{0^{\prime}}[0-800], m V$ vs. NHE & 800 & 370 \\
\hline$\Gamma\left[10^{-13}-10^{-12}\right], \mathrm{mol}$ & $1.4 \times 10^{-13}$ & $4.3 \times 10^{-13}$ \\
\hline
\end{tabular}

We could not satisfactorily fit experimental data for WT and M468Q in their AR forms using the kinetic Scheme 1 even letting all the parameters float within a wide range. This is primarily due to the lower limit on the $\mathrm{T} 1$ site reduction potential (the lowest value is set to the experimental value in solution). Since the potential of the primary electron acceptor site in the AR forms is the twoelectron reduced TNC and it has much lower reduction potential than the potential of the T1 sites, experimental data can not be reproduced using Scheme 1 with physically meaningful potentials of the T1 site. 